\title{
Innate sensing of oxidation-specific epitopes in health and disease
}

\section{Christoph J. Binder ${ }^{1,2}$, Nikolina Papac-Milicevic ${ }^{1,2}$ and Joseph L. Witztum ${ }^{3}$}

Abstract | Ageing, infections and inflammation result in oxidative stress that can irreversibly damage cellular structures. The oxidative damage of lipids in membranes or lipoproteins is one of these deleterious consequences that not only alters lipid function but also leads to the formation of neo-self epitopes - oxidation-specific epitopes (OSEs) — which are present on dying cells and damaged proteins. OSEs represent endogenous damage-associated molecular patterns that are recognized by pattern recognition receptors and the proteins of the innate immune system, and thereby enable the host to sense and remove dangerous biological waste and to maintain homeostasis. If this system is dysfunctional or overwhelmed, the accumulation of OSEs can trigger chronic inflammation and the development of diseases, such as atherosclerosis and age-related macular degeneration. Understanding the molecular components and mechanisms that are involved in this process will help to identify individuals with an increased risk of developing chronic inflammation, and will also help to indicate novel modes of therapeutic intervention.

Hormesis

A dose-response phenomenon that is characterized by beneficial effects at low doses and toxicity at high doses.
'Department of Laboratory Medicine, Medical University of Vienna, Lazarettgasse 14, AKH BT 25.2, A-1090 Vienna, Austria.

${ }^{2}$ Research Center for Molecular Medicine of the Austrian Academy of Sciences, Lazarettgasse 14, AKH BT 25.3, A-1090 Vienna, Austria.

${ }^{3}$ Department of Medicine, University of California

San Diego, California 92093, USA.

Correspondence to C.J.B. christoph.binder@ meduniwien.ac.at
In the course of evolution, most organisms have developed mechanisms to utilize oxygen for various biological processes, such as the synthesis of biomolecules, energy production and phagocytosis. Cellular usage of oxygen leads to the constant generation of reactive oxygen species (ROS), which are mainly generated by the mitochondria. In addition, ROS are generated in peroxisomes, the endoplasmic reticulum and the plasma membrane. Within eukaryotic organisms, superoxide, peroxide and hydroxyl radicals represent the main types of ROS that have high reactivity and low stability and that actively drive many cellular reactions. The biological effects of ROS greatly depend on the amounts of ROS present, which supports the idea that cellular ROS generation has characteristics of hormesis ${ }^{1}$. Indeed, at low concentrations, ROS participate in physiological processes, such as cell renewal, cellular metabolism, proliferation and differentiation ${ }^{1-4}$. However, high ROS production during an oxidative burst is crucial for host defence to ensure microbial killing ${ }^{5}$ and the formation of neutrophil extracellular traps (NETs) ${ }^{6}$. These cellular responses and the modulation of immune responses by ROS have been recently reviewed ${ }^{1,3,4,7}$. However, both exogenous triggers, such as ultraviolet (UV) irradiation, chemical toxins and hyperthermia, and endogenous conditions, including senescence, metabolic changes and inflammation, can impair the balance between the generation and the elimination of intracellular ROS, which can lead to ROS accumulation and oxidative stress. The harmful effects that are caused by oxidative stress are typically limited by antioxidant responses, which include the induction of enzymes and proteins that eliminate ROS. However, if unresolved, oxidative stress generates cellular and extracellular damage by irreversibly modifying DNA, RNA, proteins and lipids, which ultimately leads to molecular modifications that disrupt normal functions and often results in cello death. Oxidative modifications of biomolecules also lead to the generation of secondary free radicals, which further propagate oxidative damage ${ }^{1,3,4,7}$.

Lipids, particularly phospholipids, are prime targets for oxidation owing to their abundance as important building blocks of cells, extracellular vesicles and lipoproteins. The oxidation of lipids not only alters their biological function but also results in the generation of various degradation products through a process that is known as lipid peroxidation ${ }^{8}$ (BOX 1). The newly generated products propagate the effect of oxidative stress by directly modulating cellular functions and responses such as metabolism and inflammation ${ }^{9-18}$. Furthermore, as the degradation products of oxidized lipids are often highly reactive, they modify self-molecules and thereby generate structural neo-epitopes that are recognized by receptors of the immune system. These neo-epitopes have been termed oxidation-specific epitopes (OSEs) and represent a common set of epitopes present on various oxidatively modified self-proteins and lipids ${ }^{14,19}$. OSEs, including oxidized phospholipids (OxPLs) and malondialdehyde 


\section{Box 1 | Lipid peroxidation}

Lipid peroxidation is the oxidative damage of lipids that is initiated by the removal of a hydrogen atom from the $\mathrm{CH}_{2}$ group within the double bonds of polyunsaturated fatty acids (PUFAs). The subsequent addition of oxygen radicals and the formation of lipid-peroxyl radicals, which are transformed into lipid-hydroperoxide molecules can further propagate this reaction. Both enzymatic and non-enzymatic mechanisms can lead to lipid peroxidation. The enzymatic mechanisms involve lipoxygenases, cyclooxygenases and cytochrome $\mathrm{P} 450$, and result in the generation of products with high stereo-specificity ${ }^{23,144}$. Depending on the PUFA substrate, major products of lipoxygenase oxidation are hydroxyperoxyeicosatetraenoic acids (HpETEs) hydroxyeicosatetraenoic acids (HETEs), leukotrienes, lipoxins, hydroxyoctadecadienoic acids (HODEs), hepoxylins and resolvins. Oxygenation by cyclooxygenases results in the generation of prostaglandins, prostacyclin and thromboxanes, whereas cytochrome P450 generates epoxyeicosatrienoic acids (EETs), 20-HETE, thromboxanes and prostacyclins ${ }^{27,145}$. Non-enzymatic mechanisms are mediated by free radicals that are generated by NADPH oxidases and nitric oxide synthases in the presence of transition metal ions $\left(\mathrm{Fe}^{2+}\right.$ and $\mathrm{Cu}^{2+}$ ) and result in a mixture of nonspecific stereoisomers ${ }^{23,27}$. Moreover, the enzyme myeloperoxidase can also initiate non-enzymatic lipid peroxidation through the generation of reactive oxygen species (ROS), such as $\mathrm{HOCl}$, $\mathrm{HOBr}$ and $\cdot \mathrm{NO}^{145,146}$. In contrast to products of enzymatic lipid oxidation, the products of non-enzymatic processes are considered to be more toxic and damaging to the host ${ }^{147}$. Continuous degradation of lipid-hydroxyperoxides by cyclization and fragmentation in the presence of reducing metal ions $\left(\mathrm{Fe}^{2+}\right.$ and $\left.\mathrm{Cu}^{2+}\right)$ results in the generation of highly reactive terminal degradation products such as reactive aldehydes 4-hydroxynonenal (4-HNE), malondialdehyde (MDA) and 2-( $\omega$ carboxyethyl) pyrrole (CEP) ${ }^{8,24}$. Lipid peroxidation products that are generated by both mechanisms modulate a multitude of physiological processes such as cell signalling, wound healing, immune tolerance, skin barrier function, coagulation, vasodilation, modulation and the resolution of inflammation ${ }^{12,13,16,23,145,148,149}$. They are rapidly removed in healthy tissues but accumulate in many pathological conditions in which they can cause adverse effects.

Oxidation-specific epitopes (OSEs). Novel epitopes that form as a result of lipid peroxidation in response to various physiological and pathological situations. These epitopes are enriched on oxidized low-density lipoprotein and on membranes of microvesicles and apoptotic cells. They are recognized by humoral and cellular immune responses.

\section{Microvesicles}

Small $(0.1-1 \mu \mathrm{m})$ membrane vesicles shed from activated or dying cells. They expose phosphatidylserine and carry surface markers of their parental cells. They mediate the transfer of proteins, nucleic acids and lipids between cells. Elevated levels of circulating microvesicles have been reported in acute and chronic inflammatory diseases.
(MDA)-modified amino groups, have been documented on the surface of apoptotic cells, microvesicles and damaged structures such as oxidized low-density lipoproteins (OxLDLs) ${ }^{14,17,20}$. They are sensed by humoral and cellular components of the innate immune system, which mediate their removal and prevent inflammatory effects. However, if not efficiently cleared, they can also act as damage-associated molecular patterns (DAMPs) and can trigger sterile inflammation ${ }^{21,22}$ (BOX 2).

In this Review, we discuss the role of OSEs in health and disease, and the innate immune responses that specifically target OSEs as markers of oxidative stress.

\section{The generation of OSEs}

Oxidized lipids, and their degradation products, can interfere with the normal function of proteins and lipids. However, a unique aspect of some of these lipid derivatives is their ability to modify proteins and lipids and to form OSEs, which are then recognized by the immune system in a hapten-specific manner; for example, the same OSE can be present on different proteins and can be recognized by a common set of innate immune receptors. Several mechanisms can lead to the oxidation of lipids, particularly of polyunsaturated fatty acids (PUFAs) ${ }^{8,23,24}$, and important insights into the generation of OSEs have been obtained from studies on the biological activities of OxLDL that are central to the pathogenesis of atherosclerosis ${ }^{25,26}$. Lipid peroxidation involves both enzymatic mechanisms, including lipoxygenases, cyclooxygenases and cytochrome p450, and non-enzymatic mechanisms, which are mediated by free radicals and which require catalysis by transition metals or hemin ${ }^{23,26-28}$ (BOX 1). Moreover, there is now ample evidence that during apoptosis membrane lipids undergo oxidation, which results in the generation of OSEs. The exact mechanisms that lead to the oxidation of cellular membrane lipids are still being elucidated ${ }^{17,29}$.

PUFA-containing phospholipids, such as the major membrane phospholipid phosphatidylcholine, are particularly prone to oxidative damage, and their peroxidation results in the generation of a complex mixture of OxPLs and terminal degradation products ${ }^{8,23,24}$ (BOX 1). These, in turn, form adducts with, for example, the amino group of lysine residues or aminophospholipids to generate OSEs. In particular, the oxidation of the PUFA chain at the $s n-2$ position of phosphatidylcholine results in its fragmentation and the generation of reactive PUFA breakdown products such as MDA and 4-hydroxynonenal (4-HNE), which can modify self-molecules and form OSEs (FIG. 1). OSEs can also be generated by the modification of proteins with truncated phospholipids, such as oxidized phosphatidylcholine, oxidized cardiolipin (OxCL), oxidized phosphatidylserine (OxPS) and oxidized phosphatidylethanolamine $(\mathrm{OxPE})^{8,30-32}$. Furthermore, 2-( $\omega$-carboxyethyl) pyrrole (CEP) represents an adduct between (E)-4-hydroxy-7-oxohept-5-enoic acid - an oxidative fragment of docosahexaenoic acid - and the amino groups of lysines or aminophospholipids ${ }^{24,33,34}$. Notably, some of these breakdown products, such as MDA and 4-HNE, have been studied as prototypical markers of oxidative stress and can be measured by the frequently used 2-thiobarbituric acid reaction (TBAR) method $^{8}$. As a vast diversity of OxPLs can be generated, many more OSEs derived from these may exist ${ }^{23,28}$. Moreover, the oxidation of other lipids, such as cholesterol and cholesteryl esters, can lead to structural changes and altered biological activities, but their ability to be recognized by innate immune receptors is still being characterized ${ }^{10,25,35}$. It will be important to elucidate the exact structures of the relevant OSEs to better understand their recognition by the immune system. Owing to their biological activities, these products of lipid peroxidation have been implicated in a wide variety of physiological and pathological processes, and immune responses that target these products can modulate many of these effects ${ }^{14,30,36}$.

\section{Immune recognition of OSEs}

OSEs have important roles in physiological processes, as they mark oxidatively modified endogenous molecules, such as proteins and/or lipoproteins and apoptotic cells, as being damaged by increased oxidative stress. This facilitates their removal by the housekeeping functions of the immune system to maintain homeostasis. In pathological situations - in which oxidative events are greatly increased and the homeostatic functions of innate immunity are overwhelmed and/or impaired the accumulation of OSEs leads to sterile inflammation, which is a mechanism that should ultimately restore normal tissue integrity ${ }^{22}$ (BOX 2). If this fails as a result of persistent tissue damage and/or impaired resolution, the 


\section{Box 2 | Sterile inflammation}

Sterile inflammation is an inflammatory process that is elicited in response to damage-associated molecular patterns (DAMPs), which are released locally in response to tissue damage ${ }^{22}$. DAMPs are intracellular and extracellular host-derived molecules that are not usually sensed by the immune system but that are released or become modified into altered self-molecules upon tissue damage ${ }^{21}$. In analogy to pathogeninduced inflammation, sterile inflammation is triggered by the activation of the innate immune response through the recognition of DAMPs by pattern recognition receptors (PRRs), resulting in the enhanced secretion of cytokines and chemokines. Membranebound PRRs, such as Toll-like receptors (TLRs), and intracellular PRRs, such as the inflammasome, are key mediators of sterile inflammation. Cytokines belonging to the interleukin-1 (IL-1) family have been proposed to be important drivers of sterile inflammation ${ }^{150}$. Increased cytokine and chemokine secretion at the site of initial damage ultimately results in an enhanced recruitment of immune cells, such as neutrophils and macrophages. The resolution of sterile inflammation should lead to tissue repair and the re-establishment of homeostasis. Unresolved sterile inflammation is implicated in the development of several medical conditions, such as gout, Alzheimer disease, rheumatoid arthritis and atherosclerosis.

\section{Oxidized low-density}

lipoproteins

(OxLDLs). Low-density

lipoprotein (LDL) particles

(20-25nm) are the major

transporters of cholesterol in the

circulation. LDL particles consist

of $45 \%$ cholesterol, $20 \%$

phospholipids, $10 \%$ triglycerides and $25 \%$ protein. LDL can be

oxidatively modified by enzymatic and non-enzymatic mechanisms that result in many different changes to the lipid and protein moiety of LDL. Unlike LDL, OxLDL is immunogenic and pro-inflammatory, and can be recognized by macrophage scavenger receptors.

Damage-associated molecular patterns (DAMPs). Intracellular and extracellular components that are typically hidden from recognition by the immune system until cellular damage occurs. Once released into the extracellular environment they trigger sterile inflammation. Prototypical examples include high mobility group box 1 protein (HMGB1), heat shock proteins (HSPs), uric acid, mitochondrial proteins, DNA RNA and cholesterol crystals.

2-thiobarbituric acid reaction

(TBAR). The TBAR method is used to measure lipid peroxidation products, such as malondialdehyde (MDA) or other reactive aldehydes, as these products generate thiobarbituric acid-reactive substances that can be detected by colorimetric or fluorometric measurements. accumulation of OSEs can trigger chronic inflammation. Therefore, OSEs have important roles in both the regulation of tissue homeostasis and inflammatory responses (FIG. 2). The detailed characterization of OSEs and the immune responses against these OSEs have shown that they are recognized by both cellular and soluble pattern recognition receptors (PRRs), including natural IgM antibodies $^{14,31}$ (TABLE 1).

OSE sensing by cellular PRRs. OSEs are recognized by a wide variety of cell surface receptors (TABLE 1), most of which are expressed by macrophages. Macrophages mediate the uptake and phagocytosis of oxidatively altered molecules and cells, and macrophages also sense the accumulation of OSEs in the local microenvironment, which triggers signalling pathways that induce the secretion of inflammatory chemokines and cytokines $^{14,17,31}$.

Scavenger receptors represent the prototypical class of innate receptors for OSEs ${ }^{37}$. They are multi-ligand receptors that bind both modified self-structures and non-self molecules. Different types of scavenger receptors have been identified, including CD36, SRA1, SRA2, SRB1, CD68 and lectin-like oxidized LDL receptor 1 (LOX1; also known as OLR1) ${ }^{37}$. Among these, SRA1, SRA2 and CD36 are considered to be responsible for most of the OxLDL uptake by macrophages in in vitro assays, because macrophages that are deficient in CD36, SRA1 and SRA2 show 75-90\% decreased binding and degradation of $\mathrm{OxLDL}^{38}$. Detailed structural studies have shown that OxPL is a high-affinity ligand that mediates OxLDL binding to CD36 (REF. 39), and, specifically, that this is mediated by the phosphocholine (PC) headgroup of OxPL (PC-OxPL) but the PC of unoxidized phospholipids does not serve as a ligand ${ }^{40}$. Binding can also be mediated by the truncated $s n-2$ acyl chain of OxPLs $s^{41,42}$, which indicates that other classes of oxidized phospholipids such as OxPS can bind to CD36 (REFS 43,44). Similarly, PC-OxPL also mediates the uptake of OxLDL by SRB1 on macrophages ${ }^{45}$. Examples of other OSEs that are recognized by scavenger receptors include CEP-modified proteins, which are recognized by CD36 (REF. 46) and MDA-modified proteins, which are taken up by SRA1 and SRA $2^{47,48}$. A recent study showed that the endothelial scavenger receptor LOX1 mediates MDA-induced nitric oxide (NO) production ${ }^{49}$ and this receptor also binds $4-\mathrm{HNE}^{50}$.

Toll-like receptors (TLRs) also recognize and respond to OSEs. Macrophages stimulated with oxidized 1-palmitoyl-2-arachidonyl-sn-glycero-3-phosphocholine (OxPAPC) secrete interleukin-6 (IL-6), whereas IL-6 production is absent in macrophages that lack TLR4 or TIR-domain-containing adaptor protein inducing interferon- $\beta$ (TRIF; also known as TICAM1) ${ }^{51}$. Sensing of PC-OxPL seems to be important for the TLR4-mediated effects, as the PC-specific IgM T15/E06 inhibited IL-6 secretion induced by OxPAPC but did not inhibit IL- 6 secretion induced by lipopolysaccharide (LPS ${ }^{51}$. OxPLs have also been reported to stimulate macrophages via a TLR2 pathway ${ }^{52}$. Moreover, chemokine secretion by macrophages stimulated with OxLDL has been shown to require the cooperation of CD36 with a TLR4-TLR6 heterodimer ${ }^{53}$. Conversely, OxPLs induce apoptosis in macrophages via TLR2-TLR6 in cooperation with a CD36 ligand ${ }^{54}$. As most of these studies have used mixtures of different OxPL products, individual OxPL moieties might use TLR receptors in varying combinations to generate different signalling pathways. The common role of TLRs as sensors of OSEs is further supported by the findings that CEP adducts are bound by TLR2, but not by TLR4, on both endothelial cells ${ }^{33}$ and macrophages ${ }^{46}$. Furthermore, CEP selectively augments TLR2-TLR1 signalling in macrophages ${ }^{55}$ and has been shown to activate platelets via TLR2-TLR1 (REF. 56). TLRs have not yet been directly implicated in chemokine secretion that is induced by MDA or 4-HNE, but this process is probably also mediated by the cooperation of scavenger receptors with other signalling PRRs ${ }^{57}$. Oxidized cholesteryl esters (OxCEs) trigger pro-inflammatory macrophage responses via TLR4 and these involve non-classical signalling via spleen tyrosine kinase (SYK) ${ }^{35,58}$. Thus, some OSEs represent endogenous TLR ligands that have the capacity to trigger inflammatory responses. Notably, in endotoxin-induced inflammation, soluble OxPLs impair complex formation of TLR4 with CD14, MD2 and LPSbinding protein and thereby dampen LPS-induced responses ${ }^{23,59}$. Recently, epoxycyclopentenones that are present in OxPAPC were identified as active components responsible for the inhibitory effects of OxPAPC on the activation of specific TLRs by synthetic ligands ${ }^{60}$. An interesting property of the inflammatory responses to $\mathrm{OxPL}$ is the tight cooperation with scavenger receptors, such as CD36, which mediate recognition of OxPLs and probably also facilitate sensing and signalling by TLRs. Although OxLDL uptake via CD36 seems to be necessary for some macrophage signalling pathways, it is unknown to what extent scavenger receptor-mediated uptake of other OSEs is required for their cellular responses.

OSE sensing by soluble PRRs. Soluble PRRs include secreted versions of cellular PRR, pentraxins and proteins of the complement system ${ }^{61}$. Whereas some of 


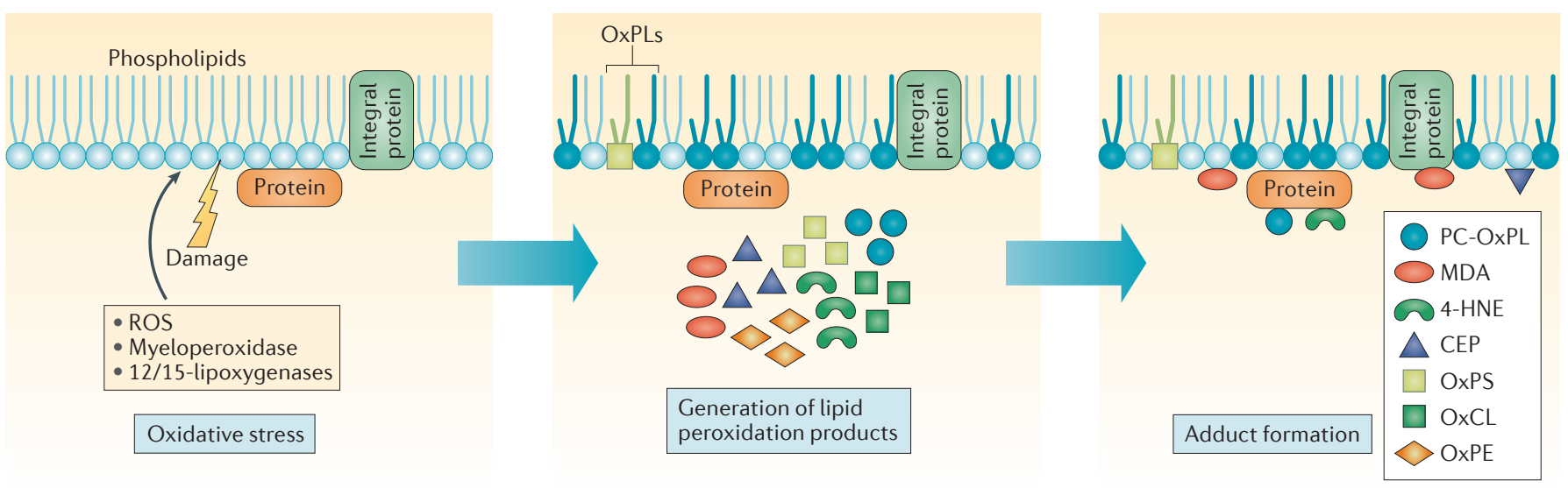

Natural IgM antibodies

Found in individuals that have not had any previous known exposure to the antigen recognized by the antibodies. They are mainly of the lgM isotype, have not undergone somatic mutations and have reactivity for many microbial pathogens and self antigens.

Scavenger receptors Cell-membrane proteins that take up oxidatively or otherwise modified low-density lipoproteins and mediate their clearance by macrophages and other cell types. They have subsequently been shown to bind a large variety of different modified proteins as well as pathogens.

\section{C-reactive protein}

(CRP). A highly conserved pentraxin that is induced and rapidly secreted by the liver in response to bacterial infections. Increased plasma CRP levels are also found in a wide variety of other non-infectious chronic inflammatory states

\section{Complement factor $\mathrm{H}$}

(CFH). A soluble glycoprotein $(155 \mathrm{kDa}$ ) that is made up of 20 short consensus repeats (SCRs) organized in a "bead on the string' manner. It is the major inhibitor of the alternative complement pathway and is highly abundant in plasma at steady-state levels of 400-700 $\mu \mathrm{g} / \mathrm{ml}$.

Figure 1 | The generation of OSEs. Tissue damage, cellular stress and cell death result in increased oxidative stress, which promotes lipid peroxidation. Lipid peroxidation can occur through non-enzymatic mechanisms, such as reactive oxygen species (ROS), and through enzymatic mechanisms, including myeloperoxidases, 12/15-lipoxygenases, cyclooxygenases and cytochrome P450. The oxidation of sn-2 polyunsaturated fatty acids (PUFAs) of membrane phospholipids leads to fragmentation and the generation of highly reactive breakdown products, such as malondialdehyde (MDA) and 4-hydroxynonenal (4-HNE) $)^{8,30-32}$. In addition, different types of oxidized phospholipids (OxPLs) can be generated from different phospholipid backbones, including oxidized phosphatidylcholine, oxidized phosphatidylethanolamine (OxPE), oxidized phosphatidylserine (OxPS) and oxidized cardiolipin (OxCL). The newly generated breakdown products and the oxidized and truncated residual core OxPLs can in turn react with free amino groups of protein side chains or lipids that are localized in their vicinity, forming stable covalent adducts and creating oxidation-specific epitopes (OSEs). Because phosphocholine (PC) as an OSE is only presented as an epitope in the context of OxPL, these epitopes are termed PC-OxPLs for clarity. The PC moiety can also be a component of the capsular polysaccharide of bacteria, where it is not part of a phospholipid and is constitutively presented as an epitope. In addition, an adduct between an oxidative fragment of docosahexaenoic acid, (E)-4-hydroxy-7-oxohept-5-enoic acid and lysines of proteins (or aminophospholipids) can lead to the formation of 2-( $\omega$-carboxyethyl) pyrrole (CEP). OSE-modified proteins or lipids are sensed by innate immune responses and represent a unique class of damage-associated molecular patterns (DAMPs).

these proteins interact with cellular PRRs to mediate the signals of bound ligands, others participate in the housekeeping functions of the host. Soluble PRRs distinguish between healthy and damaged tissues by sensing metabolic byproducts and OSEs presented on dying cells (TABLE 1).

C-reactive protein (CRP) was originally identified as the C-reactive component of plasma that could bind the PC present on the capsular polysaccharide of Streptococcus pneumoniae ${ }^{62-64}$. However, this PC moiety is not part of a phospholipid and CRP was later found to also bind PC-OxPL found on OxLDL and on apoptotic cell membranes ${ }^{65}$. This molecular mimicry between microbial $\mathrm{PC}$ and PC-OxPL enables CRP to respond to a common OSE present during both microbial infections and oxidative stress. By analogy, Porphyromonas gingivalis and group A Streptococcus have been suggested to carry MDA-like epitopes, but their ability to be recognized by MDA-specific PRRs has not been tested in detail ${ }^{66}$.

The identification of complement-regulatory protein complement factor $\mathrm{H}(\mathrm{CFH})$ as a receptor for MDAepitopes has shed important light on its function in disease $^{67}$. CFH consists of 20 short consensus repeats $(\mathrm{SCRs})^{68}$ and two of its domains - SCR7 and SCR19-20 - mediate the binding of $\mathrm{CFH}$ to $\mathrm{MDA}^{67}$. Importantly, these binding sites are hotspots for disease-associated single nucleotide polymorphisms (SNPs) ${ }^{69}$; for example, SNP rs 1061170 results in a $\mathrm{Y}>\mathrm{H}$ exchange of amino acid 402 in SCR7 and reduces the ability of plasma CFH to bind MDA by more than $65 \%$ for homozygous carriers compared with controls $s^{67}$. In addition, the use of recombinant variants of the SCR19-20 domain demonstrated that other SNPs in SCR19-20 could alter binding to $\mathrm{MDA}^{70}$. Thus, genetic variants of both MDA-binding sites of $\mathrm{CFH}$ may determine the ability of $\mathrm{CFH}$ to bind MDA. Considering the conserved structure of the regulators of complement activation, MDA-epitopes could also serve as binding sites for other members of this family ${ }^{68}$. Interestingly, the C 3 cleavage product C3a, which acts as a pro-inflammatory anaphylatoxin, was also shown to bind MDA-epitopes ${ }^{71}$.

Other soluble PRRs may recognize oxidized rather than native lipid structures on cellular surfaces, but the exact nature of these interactions is less well understood. For example, milk fat globule-epidermal growth factor 8 (MFG-E8) has recently been shown to specifically recognize $\mathrm{OxPS}^{72}$ and $\mathrm{OxPE}^{73}$. Moreover, another phosphatidylserine-binding protein, annexin A5, has been suggested to bind to OxCL and to neutralize its biological effects ${ }^{74}$.

Therefore, OSEs represent targets for several soluble PRRs of the innate immune system. As different OSEs are often present on the same surfaces, different soluble PRRs binding to them may synergize and cooperate in their effector functions. For example, apoptotic cells and microvesicles carry both MDA and PC-OxPL epitopes $^{20,75}$, each of which recruits different soluble PRRs to the same surface. 


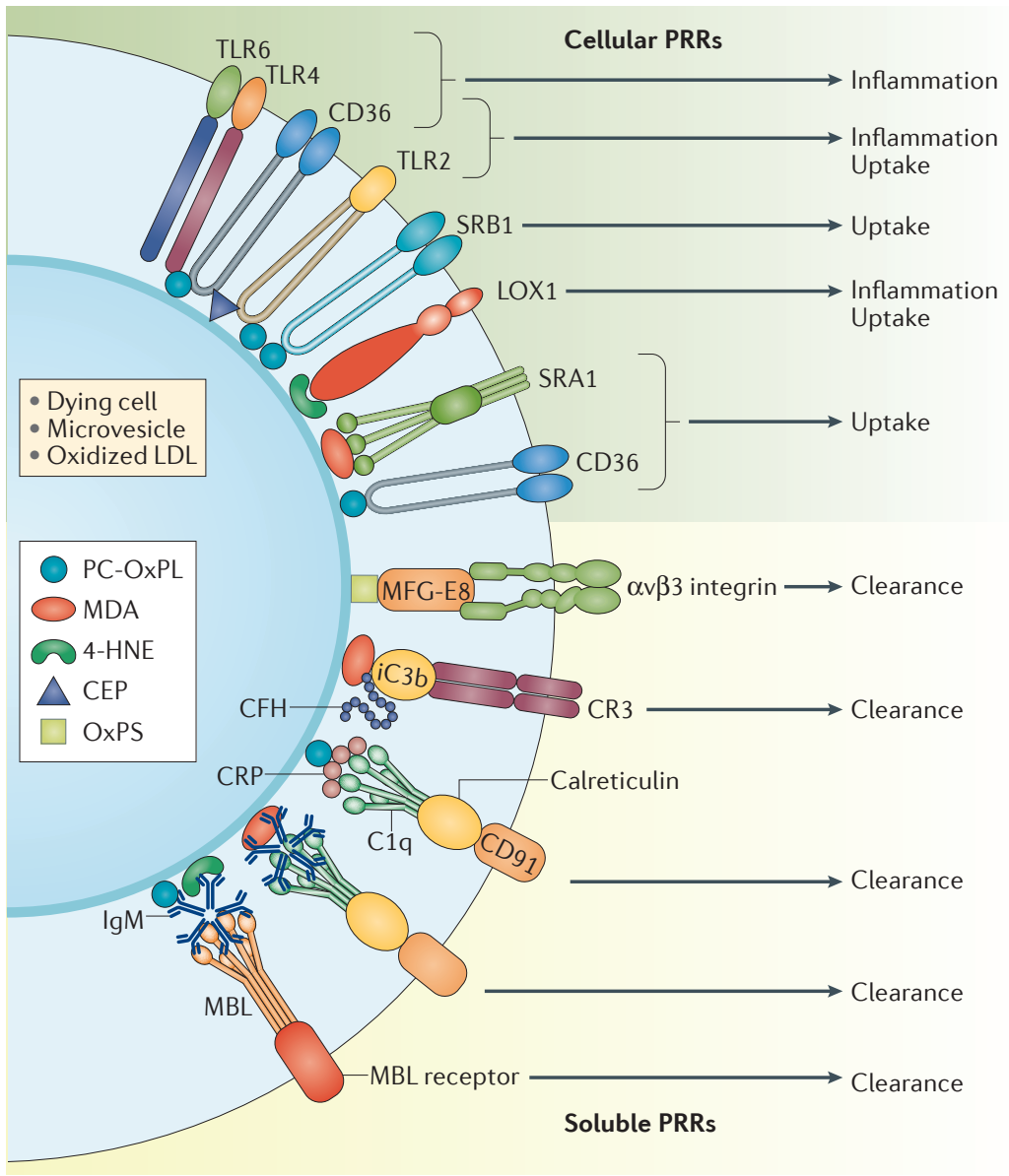

Figure 2 | Role of innate immune responses targeting OSEs. Oxidized low-density lipoproteins $(\mathrm{OxLDL})$, apoptotic cells, microvesicles and cellular debris display various types of oxidation-specific epitopes (OSEs) $)^{17,20,67,81,88}$. Components of cellular and humoral immune responses sense OSEs and mediate sterile inflammation or clearance and neutralization depending on the availability and context. Macrophages sense OSEs through various pattern recognition receptors (PRRs) that are expressed on their surface. Scavenger receptor CD36 preferentially binds and mediates the uptake of the phosphocholine (PC) of OxPL (PC-OxPL), oxidized phosphatidylserine (OxPS) and 2-( $\omega$-carboxyethyl)pyrrole (CEP). Scavenger receptor SRB1 recognizes PC-OxPL; SRA1 andlectin-like oxidized LDL receptor 1 (LOX1) bind malondialdehyde (MDA); and LOX1 recognizes 4-hydroxynonenal (4-HNE) ${ }^{14,37}$. Toll-like receptors (TLRs) mediate pro-inflammatory signals. PC-OxPL transmit inflammatory signals through a heterotrimer of TLR4-TLR6-CD36, as well as through TLR2, and CEP signals through TLR2 in cooperation with CD36 (REFS 33,46). Humoral immune responses to OSEs include natural IgM antibodies, C-reactive protein (CRP), members of complement and other soluble PRRs that are involved in the clearance of apoptotic cells. Soluble PRRs inhibit the recognition of OSEs by cellular PRRs and mediate uptake via alternative pathways. Natural IgM bound to PC-OxPL, MDA and 4-HNE epitopes are taken up by macrophages by $\mathrm{C1q}-$ calreticulinCD91-dependent or mannose-binding lectin (MBL)- and MBL receptor-dependent mechanisms. CRP bound to PC-OxPL is taken up by C1q-calreticulin-CD91-dependent mechanisms ${ }^{103,151}$. Alternative recognition of $\mathrm{C} 1 \mathrm{q}$ is mediated by $\mathrm{CD} 93$ (not shown). Complement factor $\mathrm{H}(\mathrm{CFH})$ bound to MDA provides cofactor activity for the cleavage of $\mathrm{C} 3 \mathrm{~b}$ into iC $3 \mathrm{~b}$ opsonins ${ }^{67}$ that mediate anti-inflammatory clearance via complement receptor 3 (CR3). Milk fat globule-epidermal growth factor 8 (MFG-E8) bound to OxPS or OxPE mediate clearance via av $\beta 3$ integrins ${ }^{72,73}$.

OSEs are major targets of natural IgM antibodies. OSEs have been identified as prominent antigens of natural IgM antibodies (TABLE 1). IgM antibodies in human umbilical cord blood, which represent naive natural antibodies of fetal origin, have specificity for OSEs ${ }^{75}$. In fact, titres of IgM with specificity for OxLDL and MDA-LDL were higher in cord blood samples compared with matched maternal blood samples. The specific binding properties of natural IgM antibodies have been investigated by characterizing IgM antibodies derived both from in vitro stimulated B-1 cells and from the plasma of mice lacking recombination-activating gene 1 (RAG1) that were selectively reconstituted with B-1 cells $\mathrm{s}^{75}$. These studies revealed that several OSEs, including PC-OxPL, MDA and $4-\mathrm{HNE}$ adducts are bound by up to $30 \%$ of all natural IgM found in the plasma of both wild-type and gnotobiotic mice, and by a similar percentage of IgM-secreting cells from the spleens of wild-type mice ${ }^{75}$. Furthermore, several monoclonal natural IgM antibodies with specificity for different OSEs have been characterized. For example, the well-studied PC-binding T15/E06 IgM — which contains a variable region that is composed of a canonical rearrangement of germline genes in both the $\mathrm{V}_{\mathrm{L}}$ and $\mathrm{V}_{\mathrm{H}}$ chains - was originally cloned from the spleens of atherosclerosis-prone apolipoprotein $\mathrm{E}\left(\mathrm{Apoe}^{-/-}\right)$mice as an antibody with specificity for OxLDL, which was later found to be specific for PC-OxPL ${ }^{76-78}$. The same antibody idiotype, as an IgA, was originally described due to its ability to bind PC present in the capsular polysaccharide of $S$. pneumoniae ${ }^{76,79,80}$. In addition, several other IgMs that bind different OSEs have been identified, such as the OxCL-specific LR01 and a number of others with specificity for MDA-type adducts, including E014, NA17 and LR04. Similar to T15/E06, all of the OSE-specific natural IgM antibodies that have been described exhibit germline or near-germline usage in their complementarydetermining region 3 (CDR3), identifying them as natural IgM $75,78,81,82$. Thus, natural IgM antibodies prominently participate in the response to OSEs.

\section{Role of OSEs in the maintenance of homeostasis} Physiological carriers of OSEs. Tissue homeostasis depends on the efficient removal of dying cells, which is achieved by molecular tags that mark cellular debris at different stages of cellular death. The quiescent removal of apoptotic cells is mainly mediated by phagocytes that sense several 'find-me' and 'eat-me' signals on apoptotic cells $^{83-85}$. However, in situations of increased cell death and/or inefficient clearance, apoptotic cells can release DAMPs that trigger sterile inflammation ${ }^{22}$ and render these cells immunogenic ${ }^{17}$. OSEs on late-stage apoptotic cells have been identified as important ligands for the clearance of apoptotic cells under these conditions ${ }^{17,18,75,81,86,87}$. In fact, apoptotic mammalian cells of every cellular origin tested display OSEs on their surface independently of the apoptosis trigger, and these OSEs are identical to the ones on OxLDL ${ }^{18}$ (FIG. 2). Notably, only a small proportion of early apoptotic cells display OSEs, which is in contrast to late apoptotic cells as $>50 \%$ of this cell population carries OSEs ${ }^{18,81}$. A subset of apoptotic cells is bound by natural IgM antibodies with specificity for PC-OxPL and different types of MDA epitopes, and some are bound by monoclonal antibodies with specificity for OxCL ${ }^{17,18,75,81,82}$. Furthermore, mass spectrometry has demonstrated that lipid extracts of thymocytes, in which apoptosis was induced by 
Table 1 | Cellular and humoral immune responses targeting OSEs

\begin{tabular}{|c|c|c|c|c|}
\hline \multicolumn{2}{|c|}{ Innate immune response } & \multirow{2}{*}{$\begin{array}{l}\text { OSE } \\
\text { bound }\end{array}$} & \multirow[t]{2}{*}{ Effect of OSE binding } & \multirow[t]{2}{*}{ Refs } \\
\hline Class & PRR & & & \\
\hline \multirow[t]{5}{*}{$\begin{array}{l}\text { Scavenger } \\
\text { receptors }\end{array}$} & $\begin{array}{l}\text { SRA1 and } \\
\text { SRA2 }\end{array}$ & MDA & Uptake & 47,48 \\
\hline & LOX1 & MDA & $\begin{array}{l}\text { Nitric oxide production by the } \\
\text { endothelium }\end{array}$ & 49 \\
\hline & CD36 & $\begin{array}{l}\text { - PC-OxPL } \\
\text { - OxPS } \\
\text { - CEP }\end{array}$ & $\begin{array}{l}\text { - Uptake } \\
\text { - Enhanced efferocytosis } \\
\text { - Inflammation }\end{array}$ & $\begin{array}{r}33,43 \\
44,46\end{array}$ \\
\hline & SRB1 & PC-OxPL & Uptake & 45 \\
\hline & LOX1 & 4-HNE & $\begin{array}{l}\text { - Uptake } \\
\text { - Inflammation }\end{array}$ & 50 \\
\hline \multirow[t]{3}{*}{ TLRs } & TLR4-TLR6 & PC-OxPL & Inflammation & 51,53 \\
\hline & $\begin{array}{l}\text { TLR2 and } \\
\text { TLR2-TLR6 }\end{array}$ & $\begin{array}{l}\text { - CEP } \\
\text { - OxPL }\end{array}$ & $\begin{array}{l}\text { - Inflammation } \\
\text { - Angiogenesis } \\
\text { - ER stress }\end{array}$ & $\begin{array}{r}33,46 \\
54\end{array}$ \\
\hline & Annexin A5 & $\mathrm{OxCL}$ & Neutralization & 74 \\
\hline \multirow[t]{3}{*}{ Complement } & $\mathrm{CFH}$ & MDA & $\begin{array}{l}\text { - Neutralization } \\
\text { - Opsonization (iC3b) }\end{array}$ & 67 \\
\hline & $\mathrm{C} 3 \mathrm{a}$ & MDA & Enhanced clearance of $\mathrm{C} 3 \mathrm{a}$ & 71 \\
\hline & CRP & PC-OxPL & Enhanced efferocytosis & 65 \\
\hline \multirow[t]{3}{*}{ Other PRRs } & MFG-E8 & $\begin{array}{l}\text { - OxPS } \\
\text { - OxPE }\end{array}$ & $\begin{array}{l}\text { - Enhanced efferocytosis } \\
\text { - Modulation of efferocytosis }\end{array}$ & 73,97 \\
\hline & Annexin A5 & $\mathrm{OxCL}$ & Neutralization & 74 \\
\hline & CRP & PC-OxPL & Enhanced efferocytosis & 65 \\
\hline \multirow{6}{*}{$\begin{array}{l}\text { Natural lgM } \\
\text { antibodies }\end{array}$} & LR01 & $\mathrm{OxCL}$ & Unknown & 77 \\
\hline & LR04 & MDA & Neutralization & 20 \\
\hline & NA17 & MDA & Enhanced efferocytosis & 75 \\
\hline & T15/E06 & PC-OxPL & $\begin{array}{l}\text { - Neutralization } \\
\text { - Enhanced efferocytosis } \\
\text { - Inhibition of foam cell formation }\end{array}$ & $\begin{array}{r}18,51 \\
77,78, \\
88\end{array}$ \\
\hline & E014 & MDA & Neutralization (?) & 78,89 \\
\hline & $\lg M$ & 4-HNE & Unknown & 75 \\
\hline
\end{tabular}

4-HNE, 4-hydroxynonenal; CEP, 2-( $\omega$-carboxyethyl) pyrrole; $\mathrm{CFH}$, complement factor $\mathrm{H}$; CRP, $C$-reactive protein; ER, endoplasmic reticulum; LOX1, lectin-like oxidized LDL receptor 1 ; MDA, malondialdehyde; MFG-E8, milk fat globule-epidermal growth factor 8; OSE, oxidationspecific epitope; OxCL, oxidized cardiolipin; OxPE, oxidized phosphatidylethanolamine; OxPL, oxidized phospholipid; OxPS, oxidized phosphatidylserine; PC-OxPL, phosphocholine (PC)-containing OxPL; PRR, pattern recognition receptor; SR, scavenger receptor;

TLR, Toll-like receptor. were found on fewer microvesicles but were found to a greater extent on the surface of the same microvesicles. The presence of OSEs was generally independent of cellular origin, supporting their universal presence ${ }^{20}$. Moreover, a portion of the microvesicles also carried IgM bound on their surface, which was primarily directed against MDA, consistent with their prominent presence. Interestingly, a subset of circulating microvesicles also carry CRP on their surface, which is consistent with the presence of PC-OxPL ${ }^{91}$.

Thus, different mechanisms of cell death and generation of microvesicles result in the surface presentation of various types of OSEs. An interesting area for future investigations includes understanding whether the presence of OSEs is associated with other types of cell death such as pyroptosis, necroptosis and ferroptosis ${ }^{85,92}$.

Physiological role of OSEs. In situations that are associated with increased oxidative stress, which favour OSE generation, a large amount of cellular waste is generated. For example, exposure to high light intensities in an oxygen-rich environment results in enhanced lipid peroxidation. Consequently, the retina is one of the tissues with the highest cell turnover rate, and accordingly requires high phagocytic activity for the clearance of aged photoreceptors, which are enriched in long-chain PUFAs. Both retinal pigment epithelium (RPE) and microvascular endothelial cells express several scavenger receptors that mediate the clearance of apoptotic cells, cellular debris and oxidized lipids ${ }^{93}$. Indeed, rats with a deletion variant of CD36 exhibit impaired phagocytosis of photoreceptor rod outer segments ${ }^{94}$. Whereas anionic phospholipids on apoptotic cells have been suggested to mediate this binding ${ }^{95}$, mass spectrometry studies have shown that the exposure of rat retinal cells to light results in elevated levels of OxPLs ${ }^{96}$. Importantly, OxPL inhibited the uptake of rod outer segments by wild-type cells but not by $C d 36^{-1-}$ RPE cells. OSEs may also be involved in efferocytosis via MFG-E8, which binds OxPS exposed on the surface of apoptotic cells ${ }^{72}$. MFG-E8 facilitates engulfment by phagocytes via the $\alpha v \beta 3$ and av $\beta 5$ integrin receptors, and has been shown to have an important role in the phagocytosis of photoreceptors of the outer segment ${ }^{97}$. Recent work has identified the 12/15-lipoxygenase product OxPE as a ligand for MFG-E8 with unique functional consequences in the context of apoptotic cell clearance during peritonitis ${ }^{73}$. In this study, OxPE that is exposed on the surface of alternatively activated macrophages led to the sequestration of MFG-E8, which favours the engulfment of apoptotic cells via phosphatidylserine receptors, such as $\mathrm{T}$ cell immunoglobulin and mucin domain 4 (TIM4), but blocks MFG-E8-dependent uptake of apoptotic cells by pro-inflammatory LY6C ${ }^{\text {hi }}$ monocytes. Thus, in environments with high oxidative stress, the OSE-dependent clearance of cellular debris represents a physiological housekeeping function that directs the clearance of dying cells to specific pathways.

Accumulating evidence indicates that the recognition of OSEs may also be crucial for complement-mediated clearance mechanisms, particularly of late apoptotic and 
necrotic cells, including the activity of several complement proteins and receptors, such as CRP and natural IgM, as well as pentraxin ${ }^{83,98,99}$. Natural IgM antibodies are important in apoptotic cell clearance, as mice deficient in secreted IgM are more prone to developing autoimmunity when crossed with the lupus-prone MRL/lpr background ${ }^{100}$. To date, all characterized OSE-specific natural IgM antibodies have also been found to bind apoptotic cells, and the PC-specific T15/E06 IgM and the MDA-specific NA17 antibodies enhance the uptake of apoptotic cells in mice in $v i v o^{75,101}$. Interestingly, MDA and 4-HNE accumulate in the serum of aged MRL/lpr lupus-prone mice ${ }^{102}$. MDA and PC-OxPL have also been suggested to be dominant drivers in IgM-C1q-mediated efferocytosis by dendritic cells ${ }^{87}$. Moreover, the administration of T15/E06 protected arthritis-prone DBA1 mice from collagen-induced arthritis ${ }^{86}$, suggesting an anti-inflammatory potential of neutralizing PC-OxPL. As dying cells display different OSEs at the same time, IgM with different OSE specificity may have functionally additive effects. Similarly, CRP, which also binds apoptotic cells via PC-OxPL, enhances the clearance of dying cells in a complement-dependent manner ${ }^{103}$. Although CRP and IgM are potent activators of complement, their binding on apoptotic cell surfaces does not lead to terminal complement activation because this is limited by the recruitment of the soluble regulators $\mathrm{CFH}$ and $\mathrm{C} 4$ binding protein $(\mathrm{C} 4 \mathrm{BP})^{104}$. Importantly, recruitment of $\mathrm{CFH}$ to apoptotic blebs and to necrotic RPE cells can be mediated by $\mathrm{MDA}^{67}$ and other previously described ligands ${ }^{105}$. This might allow $\mathrm{CFH}$ to provide cofactor activity for factor I on dying cells to inactivate C3b into iC3b. The phagocytosis of dying cells that have been opsonized with $\mathrm{iC} 3 \mathrm{~b}$ can inhibit nuclear factor- $\kappa \mathrm{B}(\mathrm{NF}-\kappa \mathrm{B})$ transcription, and can thereby promote anti-inflammatory clearance by phagocytes ${ }^{106}$. Notably, CFH mediates cofactor activity on MDA-decorated surfaces, and MDA binding does not interfere with this process. Moreover, owing to its reduced MDA-binding capacity, the $\mathrm{H} 402$ variant of $\mathrm{CFH}$ displayed significantly impaired cofactor activity compared with the Y402 variant, which may result in the less efficient clearance of MDA-decorated structures and increased inflammation ${ }^{67,107}$. Interestingly, $\mathrm{CFH}$ was reported to slow down efferocytosis in vitro ${ }^{104,108}$, which is beneficial in circumstances that require decreased release of ROS, nitrogen intermediates and lysosomal enzymes by phagocytes ${ }^{108,109}$. During this process, additional complement-independent neutralizing functions of CFH and OSE-specific IgM may also be crucial to prevent potential pro-inflammatory effects of the OSEs that have not yet been cleared. For example, CFH blocks MDA-induced CXC-chemokine ligand 8 (CXCL8) and CXCL1 production in vitro and in vivo, respectively ${ }^{67}$.

Similarly, the MDA-specific IgM LR04 and the PC-specific IgM T15/E06 inhibited microvesicleinduced CXCL8 secretion in human monocytes and the activation of endothelial cells, respectively ${ }^{20,88}$. These findings provide insights into the important physiological function of OSE-specific immune responses in the recognition and neutralization of cellular debris in the circulation.
In summary, OSEs mark dying cells and their cellular debris and distinguish them from viable and healthy tissues. This enables specific humoral immune responses such as natural IgM antibodies to coordinate the safe disposal of dying cells with a regulated activation of the early steps of the complement cascade. In addition, scavenger receptors that bind to OSEs mediate efferocytosis. However, if the enhanced generation of dying cells exceeds the clearance capacity of available phagocytes, the OSEs present on the accumulating apoptotic cells mediate pro-inflammatory signals and may promote autoimmunity.

\section{Innate sensing of OSEs in disease}

During acute inflammatory conditions OSEs can trigger sterile inflammation to ultimately restore tissue integrity. However, under certain conditions, the balance between the generation and the clearance of OSEs by innate immune functions is lost owing to the increased generation of OSEs and/or dysfunctional innate immune responses, which leads to increased or chronic inflammation.

OSEs have been documented in diseased tissues of patients with, for example, infectious and sterile acute lung injury, atherosclerosis, hepatitis, age-related macular degeneration (AMD), multiple sclerosis and Alzheimer disease $^{31}$. The investigation of these diseases has provided important insights into the functional role of OSE-specific immunity in both acute and chronic inflammatory models.

Role of OSEs in acute inflammation. The OSE CEP transiently accumulates during acute inflammation in a mouse model of wound healing, and induces angiogenesis in endothelial cells in a TLR2MYD88-dependent manner to stimulate wound healing and to protect against hindlimb ischaemia ${ }^{33,46}$. In this context, F4/80 hi and alternatively activated macrophages scavenge and degrade accumulated CEP in a process that requires binding of both TLR2 and CD36 (REF. 46). Notably, CEP accumulates in ageing tissues, which may lead to adverse consequences.

OSEs also accumulate in a wide variety of acute situations $\mathrm{s}^{33,59,110}$. They accumulate in the lungs of mice following acute lung injury induced by sterile acid aspiration, as well as following infection with the avian influenza virus H5N1 (REF. 51). The pulmonary lavage fluid of injured mice contains OxPL that stimulates the production of IL- 6 in macrophages, which can be ameliorated by the natural IgM antibody T15/E06. Administration of OxPL promoted acute lung injury in mice in a TLR4- and TRIF-dependent manner. In addition to PC-OxPL, MDA adducts also formed in the lungs of these mice ${ }^{51}$, and intranasal administration of MDA-modified bovine serum albumin (BSA) induced CXCL1 secretion and neutrophil recruitment into the lungs ${ }^{111}$. This is consistent with the capacity of MDA-adducts to trigger chemokine secretion in macrophages, and further demonstrates the pro-inflammatory potential of OSEs in vivo. Notably, both PC-OxPL and MDA have been documented in the lung tissues 
of patients who have died of H5N1 and SARS infections and in mice infected with lethal pathogens such as H5N1, Bacillus anthracis, SARS coronavirus and Yersinia pestis ${ }^{51}$.

Role of OSEs in atherosclerosis. Atherosclerosis is the prototypical chronic inflammatory disease in which the innate immune responses to OSEs are involved (FIG. 3). It is characterized by the deposition of LDL in the intima of large and medium-sized arteries, and the LDL subsequently undergoes oxidation to generate OxLDL. OSEs on OxLDL not only mediate uptake by macrophages to generate foam cells but also mediate many of the chronic inflammatory events that lead to the development of plaques. If plaques rupture, they can trigger thrombus formation, resulting in severe clinical manifestations such as myocardial infarction and stroke ${ }^{112}$.

PC-OxPL, MDA, 4-HNE, OxCE and CEP have all been documented in atherosclerotic lesions in humans and in animal models of disease $\mathrm{e}^{46,76,113-115}$. Endothelial cells and macrophages - which express different sets of scavenger receptors and TLRs - are major cellular sensors of OSEs in atherosclerosis. OxPLs induce the expression of chemoattractants, such as CC-chemokine ligand 2 (CCL2; also known as MCP1), fibronectin containing connecting segment 1, CXCL8 and P-selectin, and also trigger monocyte binding to endothelial cells ${ }^{23}$, which is partly mediated by TLR4 (REF. 116). 4-HNE also induces ERK1/2 and NF- $\kappa B$ activation in endothelial cells via LOX1 (REF. 50). LOX1 deficiency results in decreased lesion formation in $\mathrm{Ldlr}^{-/-}$mice ${ }^{117}$. CEP may also trigger a pro-atherogenic endothelial cell response via TLR2 (REF. 33). The expression of TLR2 is increased in endothelial cells at sites of disturbed blood flow, and TLR2 deficiency in non-haematopoietic tissues reduces atherosclerosis in $\mathrm{Ldlr}^{-/}$mice ${ }^{118}$. Thus, OSE sensing by endothelial cells is a key response in the development of atherosclerosis. Moreover, a recent study demonstrated that CEP accelerates platelet activation and thrombus formation in hyperlipidaemic $A p o e^{-/-}$mice in a TLR2-dependent manner ${ }^{56}$, indicating a role for OSEs in triggering the clinical events that result from atherosclerosis.

A hallmark of atherosclerotic lesions is the formation of lipid-laden macrophages, known as foam cells, which occurs because of the enhanced uptake of OxLDL mediated by OSE binding to scavenger receptors, such as CD36 and SRA1 (REF. 119). The enhanced uptake of cholesterol-rich LDL and subsequent inefficient removal of intracellular cholesterol can lead to a situation of supersaturation of cholesterol, leading to the formation of cholesterol crystals that damage lysosomes and that activate the NOD-, LRR- and pyrin domaincontaining protein 3 (NLRP3) inflammasome, resulting in IL-1 $\beta$ secretion $^{120}$. Indeed, cholesterol loading of macrophages with OxLDL induces IL- $1 \beta$ secretion, and $\mathrm{Ldlr}^{-/-}$mice reconstituted with NLRP3-deficient bone marrow develop less atherosclerosis ${ }^{120}$. Moreover, deficiency of CD36 and/or SRA1 in $\mathrm{Apoe}^{-/-}$and $\mathrm{Ldlr}^{-/}$ mice, respectively, reduces the size and/or complexity of lesions compared with control mice ${ }^{119}$. In addition, binding of OxLDL to CD36 induces chemokine expression and inflammasome priming through NF- $\mathrm{kB}$ activation, which is dependent on heterotrimer formation with TLR4-TLR6 (REFS 53,121). TLR4 signalling is important, as $\mathrm{Tlr} 4^{-/-}$Apoe ${ }^{-/-}$mice exhibit significantly reduced lesion size ${ }^{122}$, and clinical studies have found an SNP in TLR4, which results in impaired signalling, to be associated with reduced plaque burden and acute coronary events ${ }^{123,124}$. These studies demonstrate that sensing of OSEs, and in particular PC-OxPL, by CD36 is central to several pro-atherogenic responses in macrophages. OxLDL, as well as excessive foam cell formation, induces macrophage apoptosis, which is observed in advanced atherosclerotic lesions ${ }^{125}$. As a result of inefficient efferocytosis, apoptotic macrophages accumulate, which results in increased plaque necrosis and long-term atherosclerotic burden. Several studies demonstrate that deficiencies in certain proteins or receptors that mediate efferocytosis enhance lesion formation in atherosclerosis-prone mice ${ }^{125}$. Furthermore, the accumulation of apoptotic macrophages within lesions, and their released microvesicles, which all bear OSEs, may compete with both OxLDL and apoptotic cells for clearance by macrophages and thereby further impair efferocytosis.

Humoral immune responses are increasingly being recognized as important modulators of atherogenesis ${ }^{126}$. Although the ability of CRP to bind OSEs and to mediate apoptotic cell clearance suggests a role in atherosclerosis, there is no real support for an active role of CRP itself in the pathogenesis of the disease from animal or human studies ${ }^{127,128}$. However, CRP is an excellent biomarker of inflammation, and increased levels of CRP independently predict manifestations of atherosclerosis $^{64}$. By contrast, natural IgM antibodies clearly modulate atherosclerotic lesion formation, as deficiency in secreted IgM promotes atherosclerosis in $\mathrm{Ldlr}^{-/-}$ mice ${ }^{126}$. As many natural IgM antibodies bind OSEs ${ }^{75}$, their atheroprotective function may be mostly mediated by OSE-specific IgM, which includes housekeeping mechanisms and other protective effects. For example, T15/E06 IgM neutralizes the pro-inflammatory effects on endothelial cells and macrophages of late apoptotic cells and/or blebs carrying PC-OxPL ${ }^{17,51,88}$. T15/E06 IgM antibodies have also been shown to block the binding and uptake of OxLDL in macrophages, preventing foam cell formation ${ }^{77}$. Importantly, raising plasma levels of T15/E06 IgM by immunization with PC-containing pneumococcal extracts or passive infusion decreased atherosclerosis in $\mathrm{Ldlr}^{-1-}$ mice and vein graft atherosclerosis in $A p o e^{-/-}$mice, respectively ${ }^{129,130}$. In analogy to PC-OxPL, MDA-epitopes also trigger tumour necrosis factor (TNF) and CXCL8 in monocytes and macrophages ${ }^{67}$. In addition, the numbers of MDApositive microvesicles substantially increased at the culprit lesion sites of patients suffering from an acute coronary event compared with the number found in peripheral circulation, which could further propagate inflammation ${ }^{20}$. Thus, OSE-specific IgM can protect by neutralizing the pro-inflammatory effects of OSEs in the context of OxLDL, microvesicles and apoptotic 


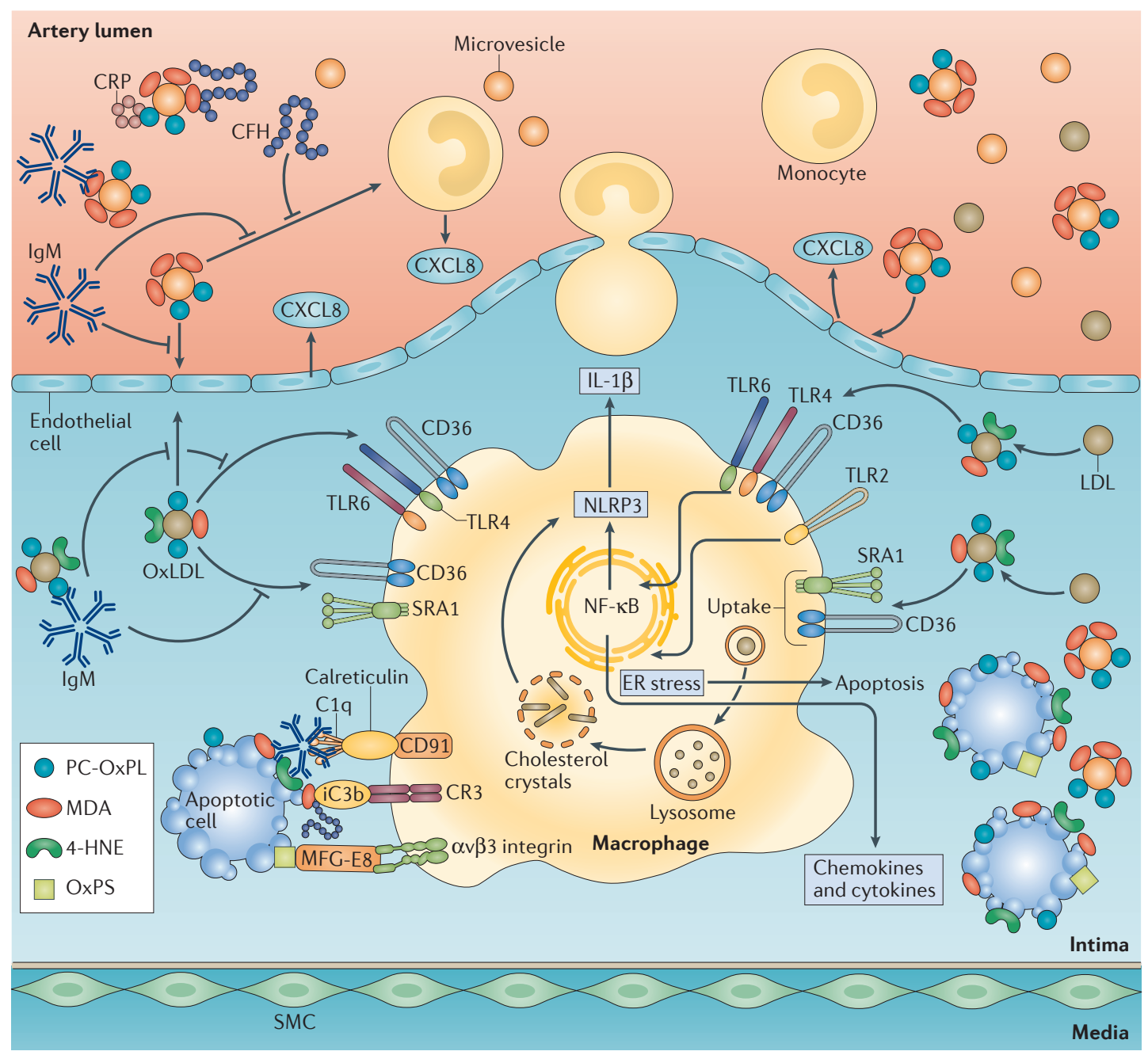

Figure 3 | Sensing of OSEs in atherosclerosis. Endothelial cells sense oxidation-specific epitopes (OSEs) present on microvesicles and oxidized low-density lipoprotein $(\mathrm{OxLDL})^{20114}$. This results in the expression of adhesion molecules and the secretion of chemoattractants leading to monocyte recruitment to the intima of the artery wall. Macrophages internalize OxLDL via scavenger receptors such as scavenger receptor A1 (SRA1), lectin-like oxidized LDL receptor 1 (LOX1), SRB1 and CD36 (REF. 37), and in cooperation with Toll-like receptor 2 (TLR2) and TLR4-TLR6 receive pro-inflammatory signals from OSEs. The enhanced uptake of OxLDL via scavenger receptors leads to the excess accumulation of intracellular cholesterol and the formation of lipid-laden foam cells, as well as the secretion of cytokines and chemokines. Excessive free cholesterol accumulation induces cholesterol crystal formation that triggers lysosome rupture and activation of the NOD-, LRR- and pyrin domain-containing protein 3 (NLRP3) inflammasome ${ }^{120}$ primed by pro-inflammatory OSE-induced signalling. Free intracellular cholesterol also induces endoplasmic reticulum (ER) stress, leading to macrophage apoptosis. As a consequence of this, and impaired efferocytosis, late-stage apoptotic cells accumulate, contributing - together with OxLDL and microvesicles - to a growing pool of OSEs inside the plaque. Natural IgM specific for OSEs blocks scavenger receptor-mediated uptake and neutralizes the pro-inflammatory effects of OSEs by promoting their complement-dependent clearance. Complement factor $\mathrm{H}$ (CFH) blocks the pro-inflammatory effects of malondialdehyde (MDA) and facilitates the anti-inflammatory clearance of MDA-decorated surfaces through Factor I-dependent iC 3 b generation ${ }^{67}$. The milk fat globule-epidermal growth factor 8 (MFG-E8) is also involved in the clearance of OSEs by recognizing oxidized phosphatidylserine (OxPS). Impaired functions of these humoral immune responses as a result of low abundance or decreased binding capacities, as well as excessive accumulation of OxLDL, microvesicles and apoptotic cells, favours the recognition of OSEs by macrophage receptors, leading to sustained inflammation. CRP, C-reactive protein; CXCL8, CXC-chemokine ligand 8; HNE, 4-hydroxynonenal; IL-1 $\beta$, interleukin-1 $\beta$; NF- $\kappa B$, nuclear factor- $\kappa B$; PC-OxPL, phosphocholine (PC) of oxidized phospholipid.

cells, and can promote the anti-inflammatory clearance of cellular debris. In line with this, several clinical studies have shown that lower levels of IgM antibodies directed against MDA-LDL and OxLDL are linked to an increased risk of cardiovascular disease (CVD) ${ }^{126}$.
Our recent identification of $\mathrm{CFH}$ as a major MDAbinding protein in plasma also suggests its potential involvement in atherosclerosis ${ }^{67}$. CFH is present in human atherosclerotic lesions, where it localizes to areas rich in MDA-epitopes ${ }^{67,131}$. Although several studies have 
Non-alcoholic steatohepatitis Characterized by lipid accumulation in the liver and inflammation, it represents a critical stage in the progression of non-alcoholic fatty liver disease to more severe potentially life-threatening conditions that are associated with liver cirrhosis and end-stage liver disease. reported an association of the $402 \mathrm{H}$ allele - which decreases binding of $\mathrm{CFH}$ to MDA - with an increased risk of heart disease and stroke, a meta-analysis of eight different study populations failed to find a significant association of this gene variant with $\mathrm{CVD}^{132}$. However, to fully understand this function of CFH in CVD, the net contribution of all SNPs that potentially affect MDA binding needs to be explored.

These data support an important role for immune responses that target OSEs in atherogenesis. OxLDL and cellular debris contribute to an excessive accumulation of OSEs, which cannot be adequately targeted by beneficial clearance mechanisms and, consequently, pro-inflammatory responses in macrophages and endothelial cells are sustained. Similarly, OSEs may promote non-alcoholic steatohepatitis ${ }^{133}$, which is increasingly being recognized as a risk factor for CVD. Studies in cholesterol-fed $\mathrm{Ldlr}^{-/-}$mice indicate that the expression of CD36 and SRA1 in Kupffer cells mediates hepatic inflammation ${ }^{134}$, whereas inducing plasma levels of the T15/E06 IgM is protective ${ }^{135}$. Consistent with these data, we recently showed that deficiency of sialic acid-binding immunoglobulin-type lectin G (Siglec-G), a negative regulator of B-1 cell function, results in increased levels of OSE-specific IgM and reduced atherosclerosis and hepatic inflammation in cholesterol-fed $\mathrm{Ldlr}^{-/-}$mice ${ }^{136}$.

OSEs in AMD. OSEs have also been implicated in $\mathrm{AMD}^{137}$, which is characterized by the accumulation of cellular waste (drusen) in the retina. Several OSEs, including PC-OxPLs, and MDA- and CEP-modified proteins, have been identified as drusen components and have been shown to trigger sterile inflammation ${ }^{138}$. For example, CEP-modified proteins induce proinflammatory gene expression and prime the NLRP3 inflammasome in monocytes via TLR2 (REFS 55,139). Drusen extracts isolated from AMD lesions activate the NLRP3 inflammasome, resulting in the secretion of IL-1 $\beta$ and IL-18 (REF. 139). Similarly, MDA-modified proteins induce the expression of CXCL8 and CXCL1 in RPE cells in in vitro and in vivo models, respectively ${ }^{67}$. Dysregulation of complement represents another major pathological driver of AMD, and genome-wide association studies have identified SNPs in genes encoding complement proteins as genetic risk factors of $\mathrm{AMD}^{69,137}$. Among these, the SNP rs 1061170 in $\mathrm{CFH}$ predisposes to increased risk for $\mathrm{AMD}^{140,141}$. Necrotic RPE cells and blebs thereof display MDA-epitopes that are recognized by $\mathrm{CFH}$, which in turn facilitates the generation of iC3b for opsonization and anti-inflammatory removal. Compared with the Y402 $\mathrm{CFH}$ variant, the cofactor activity on MDA-decorated surfaces is severely impaired when the $402 \mathrm{H} \mathrm{CFH}$ variant is used, demonstrating a functional consequence. As a result, MDA may accumulate and promote inflammation ${ }^{67}$. Indeed, transgenic mice that express the human $402 \mathrm{H}$ variant have increased susceptibility to oxidative stress-mediated injury in the retina as a result of MDA accumulation and increased inflammation ${ }^{142}$.

\section{Conclusions and future directions}

The recognition of OSEs by cellular and humoral immune responses enables the immune system to mediate important housekeeping functions, such as the removal of dying cells, cellular debris and damaged molecules. In situations of increased oxidative stress, the generation and burden of OSEs is greatly increased. As a consequence, OSEs are sensed by cellular signalling receptors, leading to the secretion of chemokines and pro-inflammatory cytokines. Thus, OSEs represent a unique class of DAMPs, which both mediate the recognition of cellular debris for housekeeping functions and, under certain circumstances, act as pro-inflammatory danger signals themselves. When this fine balance between the presence of OSEs and the capacities of the immune system to survey and respond to them is lost owing to the excessive generation of OSEs and/or their impaired removal, the development and propagation of chronic inflammatory diseases become manifest.

Although there have been a few attempts to treat patients with antioxidants to reduce the progression of CVD, these have not been successful ${ }^{143}$. These very limited studies have been criticized as premature as we currently lack sufficient knowledge to adequately design an effective therapeutic trial to test the role of oxidation in atherogenesis ${ }^{26}$. As discussed in this Review, oxidative events and the associated immune responses have beneficial effects as well as adverse consequences and we currently lack insight into the relative importance of these in vivo, which could affect the proper design of antioxidant trials.

A better understanding of the role of OSEs and OSE-reactive immune responses in the maintenance of homeostasis and in controlling inflammation are important areas of future investigations. Moreover, it is important to elucidate how different components of OSEspecific immune responses cooperate at a functional level. The identification of the genetic variants that affect some of these responses, and mechanistic studies characterizing these, may provide exciting insights into different individual predispositions to responding to oxidative stress and to developing chronic inflammation.
1. Schieber, M. $\&$ Chandel, N. S. ROS function in redox signaling and oxidative stress. Curr. Biol. 24, R453-R462 (2014).

This is an excellent review on the physiological and pathological regulation and functions of ROS.

2. Savina, A. et al. NOX2 controls phagosomal pH to regulate antigen processing during crosspresentation by dendritic cells. Cell 126, 205-218 (2006).

3. Nathan, C. \& Cunningham-Bussel, A. Beyond oxidative stress: an immunologist's guide to reactive oxygen species. Nat. Rev. Immunol. 13, 349-361 (2013).
4. Finkel, T. \& Holbrook, N. J. Oxidants, oxidative stress and the biology of ageing. Nature 408, 239-247 (2000).

5. Klebanoff, S. J. Myeloperoxidase: friend and foe J. Leukoc. Biol. 77, 598-625 (2005).

6. Almyroudis, N. G. et al. NETosis and NADPH oxidase: at the intersection of host defense, inflammation, and injury. Front. Immunol. 4, 45 (2013).

7. Bae, Y. S., Oh, H., Rhee, S. G. \& Yoo, Y. D. Regulation of reactive oxygen species generation in cell signaling. Mol. Cells 32, 491-509 (2011).
8. Esterbauer $\mathrm{H}$ Schaur, R J \& Zollner, $\mathrm{H}$ Chemistry and biochemistry of 4-hydroxynonenal, malonaldehyde and related aldehydes. Free Radic. Biol. Med. 11, 81-128 (1991).

9. Cyster, J. G., Dang E. V., Reboldi, A \& Yi, T 25-Hydroxycholesterols in innate and adaptive immunity. Nat. Rev. Immunol. 14, 731-743 (2014).

10. Spann, N. J. \& Glass, C. K. Sterols and oxysterols in immune cell function. Nat. Immunol. 14, 893-900 (2013). 
11. Bauer, J. et al. Pathophysiology of isoprostanes in the cardiovascular system: implications of isoprostanemediated thromboxane $\mathrm{A} 2$ receptor activation. Br. J. Pharmacol. 171, 3115-3131 (2014).

12. Serhan, C. N. Pro-resolving lipid mediators are leads for resolution physiology. Nature 510, 92-101 (2014).

13. Rothe, T. et al. 12/15-Lipoxygenase-mediated enzymatic lipid oxidation regulates DC maturation and function. J. Clin. Invest. 125, 1944-1954 (2015).

14. Miller, Y. I. et al. Oxidation-specific epitopes are danger-associated molecular patterns recognized by pattern recognition receptors of innate immunity. Circ. Res. 108, 235-248 (2011)

15. Kawamoto, Y. et al. Cyclopentenone prostaglandins as potential inducers of phase II detoxification enzymes. 15-deoxy- $\Delta(12,14)$-prostaglandin j2-induced expression of glutathione S-transferases. J. Biol. Chem 275, 11291-11299 (2000)

16. Clark, S. R et al. Characterization of platelet aminophospholipid externalization reveals fatty acids as molecular determinants that regulate coagulation. Proc. Natl Acad. Sci. USA 110, 5875-5880 (2013).

17. Chang, M. K. et al. Apoptotic cells with oxidationspecific epitopes are immunogenic and proinflammatory. J. Exp. Med. 200, 1359-1370 (2004).

This study demonstrated for the first time that apoptotic cells carrying OSEs have the potential to be pro-inflammatory and to trigger immune responses specific for OSEs. It also directly documented the presence of OxPLs in the membrane preparations of apoptotic, but not viable, cells by mass spectrometry.

18. Chang, M. K. et al. Monoclonal antibodies against oxidized low-density lipoprotein bind to apoptotic cells and inhibit their phagocytosis by elicited macrophages: evidence that oxidation-specific epitopes mediate macrophage recognition. Proc. Natl Acad. Sci. USA 96, 6353-6358 (1999). This is the first demonstration of the binding of apoptotic cells by natural IgM antibodies with specificity for MDA- and PC-epitopes, and a demonstration that these epitopes are important for the recognition of apoptotic cells by macrophages.

19. Chou, M. Y. et al. Oxidation-specific epitopes are important targets of innate immunity. J. Intern. Med. 263, 479-488 (2008).

20. Tsiantoulas, D. et al. Circulating microparticles carry oxidation-specific epitopes and are recognized by natural IgM antibodies. J. Lipid. Res. 56, 440-448 (2015).

21. Gallucci, S. \& Matzinger, P. Danger signals: SOS to the immune system. Curr. Opin. Immunol. 13, 114-119 (2001).

22. Chen, G. Y. \& Nunez, G. Sterile inflammation: sensing and reacting to damage. Nat. Rev. Immunol. 10, 826-837 (2010)

23. Bochkov, V. N. et al. Generation and biological activities of oxidized phospholipids. Antioxid. Redox Signal. 12, 1009-1059 (2010).

24. Salomon, R. G. Structural identification and cardiovascular activities of oxidized phospholipids. Circ. Res. 111, 930-946 (2012).

25. Harkewicz, R. et al. Cholesteryl ester hydroperoxides are biologically active components of minimally oxidized low density lipoprotein. J. Biol. Chem. 283 10241-10251 (2008).

26. Steinberg, D. \& Witztum, J. L. Oxidized low-density lipoprotein and atherosclerosis. Arterioscler. Thromb. Vasc. Biol. 30, 2311-2316 (2010)

27. O'Donnell, V. B. \& Murphy, R. C. New families of bioactive oxidized phospholipids generated by immune cells: identification and signaling actions. Blood 120, 1985-1992 (2012).

28. Lee, S. et al. Role of phospholipid oxidation products in atherosclerosis. Circ. Res. 111, 778-799 (2012)

29. Fadeel, B., Xue, D. \& Kagan, V. Programmed cell clearance: molecular regulation of the elimination of apoptotic cell corpses and its role in the resolution of inflammation. Biochem. Biophys. Res. Commun. 396 7-10 (2010)

30. Salomon, R. G., Hong, L. \& Hollyfield, J. G. Discovery of carboxyethylpyrroles (CEPs): critical insights into AMD, autism, cancer, and wound healing from basic research on the chemistry of oxidized phospholipids. Chem. Res. Toxicol. 24, 1803-1816 (2011).

31. Weismann, D. \& Binder, C. J. The innate immune response to products of phospholipid peroxidation. Biochim. Biophys. Acta 1818, 2465-2475 (2012).
32. Harnett, W. \& Harnett, M. M. Phosphorylcholine: friend or foe of the immune system? Immunol. Today 20, 125-129 (1999).

33. West, X. Z. et al. Oxidative stress induces angiogenesis by activating TLR2 with novel endogenous ligands. Nature 467, 972-976 (2010). First demonstration of CEP as a ligand for TLR2 and a demonstration of CEP in angiogenesis and wound healing involving TLR2 in vivo.

34. Wang, H. et al. 4-Hydroxy-7-oxo-5-heptenoic acid (HOHA) lactone is a biologically active precursor for the generation of 2-( $\omega$ a-Carboxyethyl)pyrrole (CEP) derivatives of proteins and ethanolamine phospholipids. Chem. Res. Toxicol. 28, 967-977 (2015)

35. Choi, S. H. et al. Polyoxygenated cholesterol ester hydroperoxide activates TLR4 and SYK dependent signaling in macrophages. PLOS ONE 8, e83145 (2013).

36. Witztum, J. L. \& Lichtman, A. H. The influence of innate and adaptive immune responses on atherosclerosis. Annu. Rev. Pathol. 9, 73-102 (2014).

37. Greaves, D. R. \& Gordon, S. The macrophage scavenger receptor at 30 years of age: current knowledge and future challenges. J. Lipid Res. 50 S282-S286 (2009)

38. Kunjathoor, V. V. et al. Scavenger receptors class A-I/II and CD36 are the principal receptors responsible for the uptake of modified low density lipoprotein leading to lipid loading in macrophages. J. Biol. Chem. 277, 49982-49988 (2002)

This paper demonstrates that SRA and CD36 are the principal receptors for the uptake of OxLDL by macrophages in vitro and in vivo.

39. Boullier, A et al. The binding of oxidized low density lipoprotein to mouse CD36 is mediated in part by oxidized phospholipids that are associated with both the lipid and protein moieties of the lipoprotein. J. Biol. Chem. 275, 9163-9169 (2000).

40. Boullier A et al. Phosphocholine as a pattern recognition ligand for CD36. J. Lipid Res. 46 969-976 (2005).

41. Li, X. M., Salomon, R. G., Qin, J. \& Hazen, S. L. Conformation of an endogenous ligand in a membrane bilayer for the macrophage scavenger receptor CD36. Biochemistry 46, 5009-5017 (2007)

42. Greenberg, M. E. et al. The lipid whisker model of the structure of oxidized cell membranes. J. Biol. Chem. 283, 2385-2396 (2008)

43. Fadok, V. A., Warner, M. L., Bratton, D. L. \& Henson, P. M. CD36 is required for phagocytosis of apoptotic cells by human macrophages that use either a phosphatidylserine receptor or the vitronectin receptor ( $\alpha$ v33). J. Immunol. 161, 6250-6257 (1998).

44. Greenberg, M. E. et al. Oxidized phosphatidylserineCD36 interactions play an essential role in macrophage-dependent phagocytosis of apoptotic cells. J. Exp. Med. 203, 2613-2625 (2006).

45. Gillotte-Taylor, K., Boullier, A, Witztum, J L. Steinberg, D. \& Quehenberger, O. Scavenger receptor class B type I as a receptor for oxidized low density lipoprotein. J. Lipid Res. 42, 1474-1482 (2001).

46. Kim, Y. W. et al. Receptor-mediated mechanism controlling tissue levels of bioactive lipid oxidation products. Circ. Res. 117, 321-332 (2015). This study describes the homeostatic property of macrophages in scavenging and controlling the tissue levels of CEP via CD36 and TLR2.

47. Duryee, M. J. et al. Scavenger receptors on sinusoidal liver endothelial cells are involved in the uptake of aldehyde-modified proteins. Mol. Pharmacol. 68, 1423-1430 (2005)

48. Shechter, I et al. The metabolism of native and malondialdehyde-altered low density lipoproteins by human monocyte-macrophages. J. Lipid Res. 22, 63-71 (1981)

49. Besler, C. et al. Mechanisms underlying adverse effects of HDL on eNOS-activating pathways in patients with coronary artery disease. J. Clin. Invest. 121, 2693-2708 (2011)

50. Kumano-Kuramochi, M. et al. Identification of 4-hydroxy-2-nonenal-histidine adducts that serve as ligands for human lectin-like oxidized LDL receptor-1. Biochem. J. 442, 171-180 (2012).

51. Imai, Y. et al. Identification of oxidative stress and Toll-like receptor 4 signaling as a key pathway of acute lung injury. Cell 133, 235-249 (2008). This study identified the pro-inflammatory properties of OxPLs as mediators of sterile and viral acute lunge injury in vivo and the crucial role of TLR4 in sensing the consequences of oxidative stress.

52. Kadl, A. et al. Oxidized phospholipid-induced inflammation is mediated by Toll-like receptor 2 . Free Radic. Biol. Med. 51, 1903-1909 (2011).

53. Stewart, C. R. et al. CD36 ligands promote sterile inflammation through assembly of a Toll-like receptor 4 and 6 heterodimer. Nat. Immunol. 11, 155-161 (2010).

This paper demonstrates that CD36 ligands, such as OxLDL and $\beta$-amyloid, facilitate TLR4 and TLR6 heterodimerization leading to a pro-inflammatory response.

54. Seimon, T. A. et al. Atherogenic lipids and lipoproteins trigger CD36-TLR2-dependent apoptosis in macrophages undergoing endoplasmic reticulum stress. Cell. Metab. 12, 467-482 (2010).

55. Saeed, A. M. et al. The oxidative stress product carboxyethylpyrrole potentiates TLR2/TLR1 inflammatory signaling in macrophages. PLOS ONE 9 e 106421 (2014).

56. Biswas, S. et al. Novel phosphatidylethanolamine derivatives accumulate in circulation in hyperlipidemic ApoE $E^{-1-}$ mice and activate platelets via TLR2. Blood http://dx.doi.org/10.1182/blood-2015-08-664300 (2016).

57. Gargiulo, S. et al. Relation between TLR4/NF-кB signaling pathway activation by 27 -hydroxycholesterol and 4-hydroxynonenal, and atherosclerotic plaque instability. Aging Cell 14, 569-581 (2015).

58. Choi, S. H. et al. Lipoprotein accumulation in macrophages via Toll-like receptor-4-dependent fluid phase uptake. Circ. Res. 104, 1355-1363 (2009).

59. Bochkov, V. N. et al. Protective role of phospholipid oxidation products in endotoxin-induced tissue damage. Nature 419, 77-81 (2002). This paper demonstrated the potential of OxPLs to inhibit LPS-induced inflammation of endothelial cells in vitro and in vivo.

60. Bretscher, P. et al. Phospholipid oxidation generates potent anti-inflammatory lipid mediators that mimic structurally related pro-resolving eicosanoids by activating Nrf2. EMBO Mol. Med. 7, 593-607 (2015)

61. Litvack, M. L. \& Palaniyar, N. Review: soluble innate immune pattern-recognition proteins for clearing dying cells and cellular components: implications on exacerbating or resolving inflammation. Innate Immun. 16, 191-200 (2010).

62. Volanakis, J. E. \& Kaplan, M. H. Specificity of C-reactive protein for choline phosphate residues of pneumococcal C-polysaccharide. Proc. Soc. Exp. Biol. Med. 136, 612-614 (1971)

63. Volanakis, J. E. Human C-reactive protein: expression, structure, and function. Mol. Immunol. 38, 189-197 (2001).

64. Verma, S., Szmitko, P. E. \& Ridker, P. M. C-reactive protein comes of age. Nat. Clin. Pract. Cardiovasc. Med. 2, 29-36 (2005)

65. Chang, M. K., Binder C. J., Torzewski, M \& Witztum, J. L. C-reactive protein binds to both oxidized LDL and apoptotic cells through recognition of a common ligand: phosphorylcholine of oxidized phospholipids. Proc. Natl Acad. Sci. USA 99, 13043-13048 (2002)

66. Turunen, S. P. et al. Recognition of Porphyromonas gingivalis gingipain epitopes by natural IgM binding to malondialdehyde modified low-density lipoprotein PLOS ONE 7, e34910 (2012).

67. Weismann, D. et al. Complement factor $\mathrm{H}$ binds malondialdehyde epitopes and protects from oxidative stress. Nature 478, 76-81 (2011) This paper shows that CFH binds to and neutralizes MDA-epitopes and describes that a genetic variant in $\mathrm{CFH}$ is associated with AMD impairs this function, offering mechanistic insights into AMD

68. Zipfel, P. F. \& Skerka, C. Complement regulators and inhibitory proteins. Nat. Rev. Immunol. 9, 729-740 (2009).

69. Zipfel, P. F., Heinen, S., Jozsi, M. \& Skerka, C Complement and diseases: defective alternative pathway control results in kidney and eye diseases. Mol. Immunol. 43, 97-106 (2006).

70. Hyvarinen, S., Uchida, K., Varjosalo, M., Jokela, R. \& Jokiranta, T. S. Recognition of malondialdehydemodified proteins by the $\mathrm{C}$ terminus of complement factor $\mathrm{H}$ is mediated via the polyanion binding site and impaired by mutations found in atypical hemolytic uremic syndrome. J. Biol. Chem. 289, 4295-4306 (2014). 
71. Veneskoski, M. et al. Specific recognition of malondialdehyde and malondialdehyde acetaldehyde adducts on oxidized LDL and apoptotic cells by complement anaphylatoxin C3a. Free Radic. Biol. Med. 51, 834-843 (2011).

72. Borisenko, G. G., Iverson, S. L., Ahlberg, S. Kagan, V. E. ¿ Fadeel, B. Milk fat globule epiderma growth factor 8 (MFG-E8) binds to oxidized phosphatidylserine: implications for macrophage clearance of apoptotic cells. Cell Death Differ. 11, 943-945 (2004)

73. Uderhardt, S. et al. 12/15-lipoxygenase orchestrates the clearance of apoptotic cells and maintains immunologic tolerance. Immunity 36, 834-846 (2012).

This study demonstrates how a 12/15-lipoxygenase-derived OSE regulates tolerogenic clearance of apoptotic cells.

74. Wan, M. et al. Oxidized but not native cardiolipin has pro-inflammatory effects, which are inhibited by Annexin A5. Atherosclerosis 235, 592-598 (2014).

75. Chou, M. Y. et al. Oxidation-specific epitopes are dominant targets of innate natural antibodies in mice and humans. J. Clin. Invest. 119, 1335-1349 (2009). This study identified OSEs as major targets of natural IgM antibodies in mice and humans and demonstrates the capacity of MDA-specific IgM to facilitate the clearance of apoptotic cells in vivo.

76. Shaw, P. X. et al. Natural antibodies with the T15 idiotype may act in atherosclerosis, apoptotic clearance, and protective immunity. J. Clin. Invest. 105, 1731-1740 (2000)

This study was the first to identify an OSE-specific IgM as a germline-encoded natural antibody with the potential to modulate atherogenesis.

77. Horkko, S. et al. Monoclonal autoantibodies specific for oxidized phospholipids or oxidized phospholipidprotein adducts inhibit macrophage uptake of oxidized low-density lipoproteins. J. Clin. Invest. 103, 117-128 (1999).

78. Palinski, W. et al. Cloning of monoclonal autoantibodies to epitopes of oxidized lipoproteins from apolipoprotein E-deficient mice. Demonstration of epitopes of oxidized low density lipoprotein in human plasma. J. Clin. Invest. 98, 800-814 (1996).

79. Potter, M. \& Lieberman, R. Common individual antigenic determinants in five of eight BALB-c IgA myeloma proteins that bind phosphoryl choline. J. Exp. Med. 132, 737-751 (1970).

80. Briles, D. E., Forman, C., Hudak, S. \& Claflin, J. L. Anti-phosphorylcholine antibodies of the T15 idiotype are optimally protective against Streptococcus pneumoniae. J. Exp. Med. 156, 1177-1185 (1982).

81. Amir, S. et al. Peptide mimotopes of malondialdehyde epitopes for clinical applications in cardiovascular disease. J. Lipid Res. 53, 1316-1326 (2012).

82. Tuominen, A. et al. A natural antibody to oxidized cardiolipin binds to oxidized low-density lipoprotein apoptotic cells, and atherosclerotic lesions. Arterioscler. Thromb. Vasc. Biol. 26, 2096-2102 (2006).

83. Arandjelovic, S. \& Ravichandran, K. S. Phagocytosis of apoptotic cells in homeostasis. Nat. Immunol. 16 907-917 (2015)

84. Poon, I. K., Lucas, C. D. Rossi, A G \& Ravichandran, K. S. Apoptotic cell clearance: basic biology and therapeutic potential. Nat. Rev. Immunol. 14, 166-180 (2014)

85. Ravichandran, K. S. Beginnings of a good apoptotic meal: the find-me and eat-me signaling pathways. Immunity 35, 445-455 (2011).

86. Chen, Y. et al. Regulation of dendritic cells and macrophages by an anti-apoptotic cell natural antibody that suppresses TLR responses and inhibits inflammatory arthritis. J. Immunol. 183, 1346-1359 (2009).

87. Chen, Y., Park, Y. B., Patel, E. \& Silverman, G. J. IgM antibodies to apoptosis-associated determinants recruit $\mathrm{C} 1 \mathrm{q}$ and enhance dendritic cell phagocytosis of apoptotic cells. J. Immunol. 182, 6031-6043 (2009). This study documents the importance of PC- and MDA-epitopes as dominant antigens on the surface of apoptotic cells in coordinating phagocytosis by dendritic cells via IgM and C1 q.

88. Huber, J et al Oxidized membrane vesicles and blebs from apoptotic cells contain biologically active oxidized phospholipids that induce monocyteendothelial interactions. Arterioscler. Thromb. Vasc. Biol. 22, 101-107 (2002).

89. Liu, M. L., Scalia, R., Mehta, J. L. \& Williams, K. J. Cholesterol-induced membrane microvesicles as novel carriers of damage-associated molecular patterns: mechanisms of formation, action, and detoxification Arterioscler. Thromb. Vasc. Biol. 32, 2113-212 (2012).

90. Rautou, P. E. et al. Microparticles, vascular function, and atherothrombosis. Circ. Res. 109, 593-606 (2011)

91. Biro, E. et al. Activated complement components and complement activator molecules on the surface of cellderived microparticles in patients with rheumatoid arthritis and healthy individuals. Ann. Rheum. Dis. 66 1085-1092 (2007)

92. Linkermann, A., Stockwell, B. R., Krautwald, S. \& Anders, H. J. Regulated cell death and inflammation: an auto-amplification loop causes organ failure. Nat. Rev. Immunol. 14, 759-767 (2014).

93. Duncan, K. G., Bailey, K. R., Kane, J. P. \& Schwartz, D. M. Human retinal pigment epithelial cells express scavenger receptors $\mathrm{BI}$ and BII. Biochem. Biophys. Res. Commun. 292, 1017-1022 (2002).

94. Sparrow, J. R., Ryeom, S. W., Abumrad, N. A. Ibrahimi, A. \& Silverstein, R. L. CD36 expression is altered in retinal pigment epithelial cells of the RCS rat. Exp. Eye Res. 64, 45-56 (1997).

95. Ryeom, S. W., Silverstein, R. L., Scotto, A. \& Sparrow, J. R. Binding of anionic phospholipids to retinal pigment epithelium may be mediated by the scavenger receptor CD36. J. Biol. Chem. 271 20536-20539 (1996).

96. Sun, M. et al. Light-induced oxidation of photoreceptor outer segment phospholipids generates ligands for CD36-mediated phagocytosis by retinal pigment epithelium: a potential mechanism for modulating outer segment phagocytosis unde oxidant stress conditions. J. Biol. Chem. 281, 4222-4230 (2006)

97. Nandrot, E. F. et al. Essential role for MFG-E8 as ligand for $\alpha \mathrm{v} \beta 5$ integrin in diurnal retinal phagocytosis. Proc. Natl Acad. Sci. USA 104, 12005-12010 (2007).

98. Peng, Y., Kowalewski, R., Kim, S. \& Elkon, K. B. The role of IgM antibodies in the recognition and clearance of apoptotic cells. Mol. Immunol. 42, 781-787 (2005)

99. Taylor, P. R. et al. A hierarchical role for classical pathway complement proteins in the clearance of apoptotic cells in vivo. J. Exp. Med. 192, 359-366 (2000)

100. Boes, M. et al. Accelerated development of IgG autoantibodies and autoimmune disease in the absence of secreted IgM. Proc. Natl Acad. Sci. USA 97, 1184-1189 (2000).

101. Ogden, C. A., Kowalewski, R., Peng, Y., Montenegro, V. $\&$ Elkon, K. B. IGM is required for efficient complement mediated phagocytosis of apoptotic cells in vivo. Autoimmunity 38, 259-264 (2005).

102. Wang, G., Li, H. \& Firoze Khan, M. Differential oxidative modification of proteins in $\mathrm{MRL}^{+/+}$and MRL/Ipr mice: Increased formation of lipid peroxidation-derived aldehyde-protein adducts may contribute to accelerated onset of autoimmune response. Free Radic. Res. 46, 1472-1481 (2012).

103. Gershov, D., Kim, S., Brot, N. \& Elkon, K. B. C-Reactive protein binds to apoptotic cells, protects the cells from assembly of the terminal complement components, and sustains an antiinflammatory innate immune response: implications for systemic autoimmunity. J. Exp. Med. 192, 1353-1364 (2000)

104. Trouw, L. A. et al. C4b-binding protein and factor $\mathrm{H}$ compensate for the loss of membrane-bound complement inhibitors to protect apoptotic cells against excessive complement attack. J. Biol. Chem. 282, 28540-28548 (2007)

105. Trouw, L. A., Blom, A. M. \& Gasque, P. Role of complement and complement regulators in the removal of apoptotic cells. Mol. Immunol. 45 1199-1207 (2008)

106. Amarilyo, G. et al. iC3b-opsonized apoptotic cells mediate a distinct anti-inflammatory response and transcriptional NF-kB-dependent blockade. Eur. J. Immunol. 40, 699-709 (2010)

107. Lauer, N. et al. Complement regulation at necrotic cell lesions is impaired by the age-related macular degeneration-associated factor-H His402 risk variant. J. Immunol. 187, 4374-4383 (2011).

108. Kang, Y. H., Urban, B. C., Sim, R. B. \& Kishore, U. Human complement factor $\mathrm{H}$ modulates $\mathrm{C} 1 \mathrm{q}$-mediated phagocytosis of apoptotic cells. Immunobiology 217 455-464 (2012).

109. Gresham, H. D et al. Negative regulation of phagocytosis in murine macrophages by the Src kinase family member Fgr. J. Exp. Med. 191 515-528 (2000)

110. Matt, U. et al. WAVE1 mediates suppression of phagocytosis by phospholipid-derived DAMPs. J. Clin. Invest. 123, 3014-3024 (2013).

111. Wyatt, T. A. et al. Malondialdehyde-acetaldehyde adducted protein inhalation causes lung injury. Alcohol 46, 51-59 (2012).

112. Libby, P., Lichtman, A. H. \& Hansson, G. K. Immune effector mechanisms implicated in atherosclerosis: from mice to humans. Immunity 38, 1092-1104 (2013)

113. Jurgens, G. et al. Immunostaining of human autopsy aortas with antibodies to modified apolipoprotein B and apoprotein(a). Arterioscler. Thromb. 13 1689-1699 (1993).

114. Yla-Herttuala, S. et al. Evidence for the presence of oxidatively modified low density lipoprotein in atherosclerotic lesions of rabbit and man. J. Clin. Invest. 84, 1086-1095 (1989).

This study was the first to document the in vivo presence of oxidized LDL and of OSEs on LDL isolated from human atherosclerotic plaques.

115. van Dijk, R. A. et al. Differential expression of oxidation-specific epitopes and apolipoprotein(a) in progressing and ruptured human coronary and carotid atherosclerotic lesions. J. Lipid Res. 53, 2773-2790 (2012).

116. Walton, K. A. et al. Receptors involved in the oxidized 1-palmitoyl-2-arachidonoyl-

sn-glycero-3-phosphorylcholine-mediated synthesis of interleukin-8. A role for Toll-like receptor 4 and a glycosylphosphatidylinositol-anchored protein J. Biol. Chem. 278, 29661-29666 (2003).

117. Mehta, J. L. et al. Deletion of LOX-1 reduces atherogenesis in LDLR knockout mice fed high cholesterol diet. Circ. Res. 100, 1634-1642 (2007).

118. Mullick, A. E. et al. Increased endothelial expression of Toll-like receptor 2 at sites of disturbed blood flow exacerbates early atherogenic events. J. Exp. Med. 205, 373-383 (2008)

119. Moore, K. J., Sheedy, F. J. \& Fisher, E. A. Macrophages in atherosclerosis: a dynamic balance. Nat. Rev. Immunol. 13, 709-721 (2013).

120. Duewell, P. et al. NLRP3 inflammasomes are required for atherogenesis and activated by cholesterol crystals. Nature 464, 1357-1361 (2010).

121. Sheedy, F. J. et al. CD36 coordinates NLRP3 inflammasome activation by facilitating intracellular nucleation of soluble ligands into particulate ligands in sterile inflammation. Nat. Immunol. 14, 812-820 (2013).

122. Michelsen, K. S. et al. Lack of Toll-like receptor 4 or myeloid differentiation factor 88 reduces atherosclerosis and alters plaque phenotype in mice deficient in apolipoprotein E. Proc. Natl Acad. Sc USA 101, 10679-10684 (2004).

123. Ameziane, N. et al. Association of the Toll-like receptor 4 gene Asp299Gly polymorphism with acute coronary events. Arterioscler. Thromb. Vasc. Biol. 23, e61-e64 (2003).

124. Kiechl, S. et al. Toll-like receptor 4 polymorphisms and atherogenesis. N. Engl. J. Med. 347, 185-192 (2002).

125. Tabas, I. Macrophage death and defective inflammation resolution in atherosclerosis. Nat. Rev. Immunol. 10 36-46 (2010).

126. Tsiantoulas, D., Diehl, C. J., Witztum, J. L. \& Binder, C. J. B cells and humoral immunity in atherosclerosis. Circ. Res. 114, 1743-1756 (2014).

127. Tanigaki, K. et al. Fc $\gamma$ receptors and ligands and cardiovascular disease. Circ. Res. 116, 368-384 (2015).

128. Zacho, J. et al. Genetically elevated C-reactive protein and ischemic vascular disease. N. Engl. J. Med. 359, 1897-1908 (2008).

129. Binder, C. J. et al. Pneumococcal vaccination decreases atherosclerotic lesion formation: molecular mimicry between Streptococcus pneumoniae and oxidized LDL. Nat. Med. 9, 736-743 (2003).

130. Faria-Neto, J. R. et al. Passive immunization with monoclonal IgM antibodies against phosphorylcholine reduces accelerated vein graft atherosclerosis in apolipoprotein E-null mice. Atherosclerosis 189 , 83-90 (2006)

131. Oksjoki, R. et al. Association between complement factor $\mathrm{H}$ and proteoglycans in early human coronary atherosclerotic lesions: implications for local regulation of complement activation. Arterioscler. Thromb. Vasc. Biol. 23, 630-636 (2003). 
132. Sofat, R. et al. Genetic variation in complement factor $\mathrm{H}$ and risk of coronary heart disease: eight new studies and a meta-analysis of around 48,000 individuals. Atherosclerosis 213, 184-190 (2010).

133. Walenbergh, S. M., Koek, G. H., Bieghs, V. \& Shiri-Sverdlov, R. Non-alcoholic steatohepatitis: the role of oxidized low-density lipoproteins. J. Hepatol. 58, 801-810 (2013).

134. Bieghs, V. et al. Internalization of modified lipids by CD36 and SR-A leads to hepatic inflammation and Iysosomal cholesterol storage in Kupffer cells. PLOS ONE 7, e34378 (2012).

135. Bieghs, V. et al. Specific immunization strategies against oxidized low-density lipoprotein: a novel way to reduce nonalcoholic steatohepatitis in mice. Hepatology 56, 894-903 (2012).

136. Gruber, S. et al. Sialic acid-binding immunoglobulinlike lectin $\mathrm{G}$ promotes atherosclerosis and liver inflammation by suppressing the protective functions of B-1 cells. Cell Rep. 14, 2348-2361 (2016)

137. McHarg, S., Clark, S. J., Day, A. J. \& Bishop, P. N. Age-related macular degeneration and the role of the complement system. Mol. Immunol. 67, 43-50 (2015).

138. Handa, J. T. How does the macula protect itself from oxidative stress? Mol. Aspects Med. 33, 418-435 (2012).
139. Doyle, S. L. et al. NLRP3 has a protective role in agerelated macular degeneration through the induction of IL-18 by drusen components. Nat. Med. 18, 791-798 (2012).

140. Haines, J. L. et al. Complement factor $\mathrm{H}$ variant increases the risk of age-related macular degeneration. Science 308, 419-421 (2005)

141. Edwards, A. O. et al. Complement factor H polymorphism and age-related macular degeneration. Science 308, 421-424 (2005).

142. Aredo, B. et al. A chimeric Cfh transgene leads to increased retinal oxidative stress, inflammation, and accumulation of activated subretinal microglia in mice. Invest. Ophthalmol. Vis. Sci. 56, 3427-3440 (2015).

143. Kris-Etherton, P. M. et al. Antioxidant vitamin supplements and cardiovascular disease. Circulation 110, 637-641 (2004).

144. Niki, E., Yoshida, Y., Saito, Y. \& Noguchi, N. Lipid peroxidation: mechanisms, inhibition, and biological effects. Biochem. Biophys. Res. Commun. 338, 668-676 (2005)

145. Thomas, C. P. \& O'Donnell, V. B. Oxidized phospholipid signaling in immune cells. Curr. Opin Pharmacol. 12, 471-477 (2012).

146. Nicholls, S. J. \& Hazen, S. L. Myeloperoxidase modified lipoproteins, and atherogenesis. J. Lipid Res. 50, S346-S351 (2009).
147. Mauerhofer, C. Philippova, M. Oskolkova, O V \& Bochkov, V. N. Mol. Aspects Med. 49, 78-90 (2016).

148. Krieg, P. et al. Aloxe3 knockout mice reveal a function of epidermal lipoxygenase- 3 as hepoxilin synthase and its pivotal role in barrier formation. J. Invest. Dermatol. 133, 172-180 (2013).

149. Chiang, N., Arita, M. \& Serhan, C. N. Antiinflammatory circuitry: lipoxin, aspirin-triggered lipoxins and their receptor ALX. Prostaglandins Leukot. Essent. Fatty Acids 73, 163-177 (2005).

150. Latz, E., Xiao, T. S. \& Stutz, A. Activation and regulation of the inflammasomes. Nat. Rev. Immunol. 13, 397-411 (2013).

151. Marnell, L., Mold, C. \& Du Clos, T. W. C-reactive protein: ligands, receptors and role in inflammation. Clin. Immunol. 117, 104-111 (2005).

\section{Acknowledgements}

The work carried out in the authors' laboratories related to this Review is supported by the Austrian Science Fund (SFB-30 and SFB-54 to C.J.B.) and the US National Institutes of Health (grants HL086559, HL119828 and HL055798 to J.L.W.) The authors thank V. Krajina for help with the illustrations.

Competing interests statement

The authors declare competing interests: see Web version for details. 